\title{
Photon position operators and localized bases
}

\author{
Margaret Hawtonl and William E. Baylisi \\ ${ }^{1}$ Department of Physics, Lakehead University, \\ Thunder Bay, Ontario, Canada P' 7 B 5E1 \\ ${ }^{2}$ Department of Physics, University of Windsor, \\ Windsor, Ontario, Canada
}

\begin{abstract}
We extend a procedure for construction of the photon position operators with transverse eigenvectors and commuting components [Phys. Rev. A 59, 954 (1999)] to body rotations described by three Euler angles. The axial angle can be made a function of the two polar angles, and different choices of the functional dependence are analogous to different gauges of a magnetic field. Symmetries broken by a choice of gauge are re-established by transformations within the gauge group. The approach allows several previous proposals to be related. Because of the coupling of the photon momentum and spin, our position operator, like that proposed by Pryce, is a matrix that does not commute with the spin operator. Unlike the Pryce operator, however, our operator has commuting components, but the commutators of these components with the total angular momentum require an extra term to rotate the matrices for each vector component around the momentum direction. Several proofs of the nonexistence of a photon position operator with commuting components are based on overly restrictive premises that do not apply here.
\end{abstract}

PACS number(s): 03.65.Ta, 14.70.Bh, 42.50.-p, 03.65.Vf

Typeset using REVTEX 


\section{INTRODUCTION}

Since the early days of quantum mechanics, it has been claimed that there is no photon position operator with commuting components, and hence that a basis of its localized eigenvectors, $\left|\mathbf{r}^{\prime}\right\rangle$, does not exist [1]. As a consequence, it is widely held that there is no coordinate-space photon wave function, $\psi\left(\mathbf{r}^{\prime}\right)=\left\langle\mathbf{r}^{\prime} \mid \psi\right\rangle$ [2]. Wave functions for photons in momentum space are commonly used, and it is there where the position operator would be defined. While it may generally be possible to use a second-quantized formalism in which the fields are operators in Fock space [2, 3], the usefulness of a coordinate-space photon wave function for describing such phenomena as photon interference is sufficiently well recognized that a number of authors have introduced versions of effective spatial wave functions 《4,5]. The existence of a position operator may also be important for the consistency of some work in the second-quantized formalism.

A number of authors [6 12] have discussed and to some extent resolved well-known problems of Lorentz covariance and causality that exist for any position operator in relativistic quantum theory. However, there are additional problems for massless particles of spin one or greater, and a number of "proofs" have been presented of the nonexistence of a photon position operator in the literature 13 18. In spite of these, one of us has recently produced a counter example: a Hermitian position operator with commuting components and localized transverse eigenvectors [19]. However, the asymmetric, singular nature of the operator was puzzling, and questions concerning its compatibility with work concluding the nonexistence of such operators were not fully addressed.

In the present paper we attempt to resolve these issues. Our principal tool is a generalization of the new position operator to include an arbitrary axial rotation, which may be a function of the other two Euler angles that parameterize the body rotation. This gener-

alization provides insight into the geometry underlying the operator and allows us to unify several previous approaches.

While position operators can be defined in several ways, the one in Ref. [19], henceforth 
to be referred to as I, was constructed by requiring its components to have eigenvectors transverse to the momentum and in the directions of the momentum-space polar unit vectors. The position operator thus obtained takes the form of a $3 \times 3$ matrix and will be referred to here as $\underline{\mathbf{r}}^{(0)}$, where the underscore denotes the matrix character and (0) refers to use of the spherical polar basis vectors $\hat{\boldsymbol{\theta}}$ and $\hat{\boldsymbol{\phi}}$. The momentum-space operator $\underline{\mathbf{r}}^{(0)}$ can be expressed by a transformation that rotates the components of the photon state function in momentum space to a fixed photon reference frame, differentiates, and then rotates back to the lab frame. Since two angles suffice to specify the direction of the photon momentum $\mathbf{p}$, the rotations require only two independent parameters.

Here we follow a more general approach by including, in addition to the polar angles $\theta$ and $\phi$, the axial Euler angle, $\chi$. Let the unit vectors along the Cartesian axes be $\mathbf{e}_{1}, \mathbf{e}_{2}$ and $\mathbf{e}_{3}$. A rotation about $\mathbf{p}$ by an angle $\chi$ permits an arbitrary choice of the transverse unit vectors $\mathbf{e}_{\mathbf{p} 1}$ and $\mathbf{e}_{\mathbf{p} 2}$, which are obtained by a rotation from the fixed unit vectors $\mathbf{e}_{1}$ and $\mathbf{e}_{2}$ in the given reference frame. The same rotation is designed to take $\mathbf{e}_{3}$ to the momentum direction $\hat{\mathbf{p}}=\mathbf{p} / p$, but $\hat{\mathbf{p}}$ depends only on the polar angles $\theta$ and $\phi$; it is independent of $\chi$. We show below that if $\chi$ is independent of $\theta$ and $\phi$, the position operator is just $\underline{\mathbf{r}}^{(0)}$ as found previously [19]. However, we are also free to choose $\chi$ to be a function $\chi=\chi_{\mathbf{p}}(\theta, \phi)$ of the polar angles. The momentum-space position operator that corresponds to such a choice is

$$
\underline{\mathbf{r}}=\underline{\mathbf{r}}^{(0)}+\nabla \chi_{\mathbf{p}}
$$

in units with $\hbar=1$. (It is understood that the second term on the rhs is multiplied by a unit $3 \times 3$ matrix. To simplify notation, we do not indicate unit matrices explicitly.) A change in the momentum-space gradient $\nabla \chi_{\mathbf{p}}(\theta, \phi)$ is analogous to a gauge transformation of $\underline{\mathbf{r}}$. The singularity previously found in $\underline{\mathbf{r}}^{(0)}$ is analogous to that of a monopole string and commonly arises in electromagnetic-like gauge potentials. For any choice of $\chi_{\mathbf{p}}, \underline{\mathbf{r}}$ has less symmetry than one would expect and under parity inversion it does not simply change its sign. However, as we show below, the symmetry is restored when one includes the full group of possible gauge transformations rather than only a single choice of gauge. 
In the case of massive particles of spin $S$, any component of the spin can be used to define an eigenstate basis. The basis functions corresponding to a space-fixed quantization direction span a rotationally invariant linear manifold and carry irreducible representations of the Poincaré group. Because the momentum-space basis states for massive particles have directions in space that are invariant under infinitesimal translations, the position operator can be identified with the Hermitian generator $i \nabla$ of such translations, where $\nabla$ is the momentum-space gradient. Since $i \nabla$ is a differential operator proportional to the unit matrix, it commutes with spin matrices that represent space-fixed components of the spin. For massless particles, on the other hand, representations of the Poincaré group are reduced to the two irreducible representations carried by helicity states in which the spin component along the momentum $\mathbf{p}$ is $\pm S$ [20]. Since the two helicity subspaces are invariant under the actions of the Poincaré group, the allowed spin states for a massless particle with $S \geq 1$ form rotationally invariant manifolds with fewer than the $2 S+1$ independent elements required for states quantized along a given spatial direction. As elaborated below (see Section VI), this is important in understanding the relevance of the nonexistence result of Newton and Wigner [13]. The basis vectors corresponding to the two allowed helicities are transverse to $\hat{\mathbf{p}}$ and therefore require a modified position operator, one that is no longer independent of the spin.

The generator of rotations is, as usual,

$$
\underline{\mathbf{J}}=-i \mathbf{p} \times \nabla+\underline{\mathbf{S}},
$$

where the vector components of $\underline{\mathbf{S}}$ are the spin-one matrices $\underline{S}_{j}$ whose explicit elements depend of the basis used for the state vectors (see discussion in the following section). The term $-i \mathbf{p} \times \nabla=\mathbf{L}$ is required in order to generate a rotation of the argument of the wave function, whereas $\underline{\mathbf{S}}$ generates the rotation of its components. Our use of the sum (2) for photons is consistent with Bargmann and Wigner's [21] proof that $\underline{\mathbf{J}}$ is the generator of rotations for a particle of arbitrary mass. 
For the position operator $\mathbf{r}$ to transform as a vector, it is traditionally required that

$$
\left[\underline{J_{j}}, r_{k}\right]=i \varepsilon_{j k l} r_{l},
$$

where repeated indices are summed over. For massive particles, this commutator follows directly from the assumptions that components of the operator $\mathbf{r}$ commute with each other and with the spin matrices, and that they satisfy the canonical commutator relations with the momentum,

$$
\left[p_{j}, r_{k}\right]=-i \delta_{j k},
$$

since then the components of either $\mathbf{L}$ or $\underline{\mathbf{J}}$ rotate one component of $\mathbf{r}$ into another:

$$
\begin{aligned}
{\left[\underline{J_{j}}, r_{k}\right] } & =\left[L_{j}, r_{k}\right]=\varepsilon_{j m n} r_{m}\left[p_{n}, r_{k}\right] \\
& =i \varepsilon_{j k m} r_{m} .
\end{aligned}
$$

Whereas this clearly applies to the position operator $i \nabla$ usually chosen for massive particles, the photon position operators proposed by Pryce [22] and one of us [19] are matrices that do not commute with the spin. As we discuss in more detail in the following section, components of the Pryce position operator satisfy the commutation relations (3) but do not commute with each other. Consequently, their eigenstates cannot have eigenvalues for more than one component of the position operator and thus cannot fully define the position of a photon.

On the other hand, our position operator has commuting components, but at the cost of the commutation relation (3). For a given functional form of $\chi_{\mathbf{p}}$, an infinitesimal rotation of $\underline{\mathbf{r}}$ results in an incremental axial rotation of the matrix $\underline{r_{j}}$ for each component by $d \chi-d \chi_{\mathbf{p}}$, and this yields the extra term derived below [see Eq. [67)]. Recent proofs of the nonexistence of a photon position operator and localized states have assumed components $r_{j}$ that satisfy Eq.(3) [17,18], thus excluding the position operators discussed here.

As in I, the position operator is constructed with the requirement that its components have transverse eigenvectors. It is related to the position operator for massive particles, whose components have eigenvectors with fixed directions in momentum space, by a transformation with the form of a spin rotation and a dilation. As discussed more fully in the 
following section, this similarity-like transformation includes a spin rotation $D(\phi, \theta, \chi)$ from fixed directions in the photon reference frame to the longitudinal and transverse directions in the lab. Because the longitudinal direction is independent of the axial angle $\chi$, a family of transformations with different functional dependencies $\chi=\chi_{\mathbf{p}}(\theta, \phi)$ are possible. We find that this family of transformations includes ones that relate the Shirokov and Lomont and Moses irreducible helicity representations to the Foldy form of the Poincaré algebra. The use of Euler angles thus gives a unified approach to the relationships among these representations and to the position operators with transverse eigenvectors obtained in the present paper.

In Section II we briefly review the Foldy and helicity representations of the Poincaré operators. We then describe the relationship of the angular momentum and boost operators to the Pryce [22] position operator. In Section III the results of paper I are reviewed and then generalized to an arbitrary functional dependence $\chi=\chi_{\mathbf{p}}(\theta, \phi)$ of the axial Euler angle on the polar angles of $\hat{\mathbf{p}}$. The Poincaré group, the relationship of the position operator to the angular momentum and to the boost operators, and the localized states are then examined in Section IV. The connection of our work to Berry's phase is treated in Section $\mathrm{V}$, and the relation of string singularities to nonintegrable angles is stressed. In Section VI, the consistency of our position operator with nonexistence proofs is addressed, and its implications for the photon wave function are briefly discussed. Details and alternative derivations of some of the geometric arguments are presented in the Appendices.

\section{FOLDY AND HELICITY REPRESENTATIONS OF THE POINCARÉ OPERATORS}

Foldy [23] wrote down the form of the ten generators of inhomogeneous Lorentz transformations (the Poincaré operators) for particles with spin $S$ and mass $m$ in the standard space-fixed representation. His operators also exist in the $m \rightarrow 0$ limit, the only case con-

sidered here, and in momentum space they comprise the momentum $\mathbf{p}$, the Hamiltonian 
$H=p c$, the total angular momentum operator $\mathbf{J}[$ Eq.(2)], and the boost operator

$$
\underline{\mathbf{K}}=i p \nabla+\hat{\mathbf{p}} \times \underline{\mathbf{S}} .
$$

(Foldy's symmetrized form of the $i p \nabla$ term was criticized by Chakrabarti [24]. The correct form is related to the momentum-space normalization weight used for scalar products, which is discussed toward the end of this section. Note that the boost generator $\mathbf{K}$ is $-\mathbf{N}$ in Chakrabarti's paper [24].)

The standard helicity representation was introduced by Lomont and Moses [25] to provide a realization of the generators for the zero-mass case. The representation is distinct from the zero-mass limit of the Foldy representation, given above, because the carrier space has been split into invariant subspaces labeled by the two helicity components $\kappa= \pm S$. Vector components of the spin $\mathbf{S}$ are referred to the photon reference frame in which the momentum direction $\hat{\mathbf{p}}$ is $\mathbf{e}_{3}$. Chakrabarti [24] showed that the zero-mass limit of the Foldy representation is related to the Lomont and Moses helicity representation by the unitary transformation

$$
\underline{O}_{\mathrm{F}}=\underline{U} \underline{O}_{\mathrm{LM}} \underline{U}^{-1}
$$

where $\underline{O}_{\mathrm{F}}$ is an operator in the Foldy representation, $\underline{O}_{\mathrm{LM}}$ is the corresponding operator in the helicity representation of Lomont and Moses, and the unitary transformation has a matrix representation $\underline{U}$ that we recognize as the spin rotation

$$
\underline{U}=\exp (-i \theta \hat{\boldsymbol{\phi}} \cdot \underline{\mathbf{S}}) .
$$

The transformation (8) rotates $S_{3}$ directly into $\mathbf{S} \cdot \hat{\mathbf{p}}$. As above, $\theta$ and $\phi$ are the usual spherical polar angles of $\mathbf{p}$ in momentum space and $\hat{\phi}$ is the unit tangent vector in the direction of increasing $\phi$. It is perpendicular to both $\mathbf{e}_{3}$ and $\hat{\mathbf{p}}$. In the transformation (8) and its inverse, it is important to recognize that the unitary operator Eq.(9) does not generally commute with the momentum-space gradient $\nabla$ because of the dependence of $\theta$ and $\hat{\boldsymbol{\phi}}$ on $\hat{\mathbf{p}}$. The Hermitian generators of the infinitesimal transformations of the Lomont and Moses helicity representation are thus given by the Foldy representation and the inverse 
of (8) to be [24] $\mathbf{p} \equiv \sum_{j} p_{j} \mathbf{e}_{j}, H=p c$,

$$
\underline{\mathbf{J}}_{\mathrm{LM}}=-i \mathbf{p} \times \nabla+\left(\frac{\hat{\mathbf{p}}+\mathbf{e}_{3}}{1+\cos \theta}\right) \underline{S_{3}},
$$

and

$$
\underline{\mathbf{K}}_{\mathrm{LM}}=i p \nabla+\left(\frac{\hat{\mathbf{p}} \times \mathbf{e}_{3}}{1+\cos \theta}\right) \underline{S_{3}}
$$

where the matrix $\underline{S_{3}}$ can be replaced by $\kappa$ when restricted to the invariant subspace of definite helicity.

A different helicity representation was obtained earlier by Shirokov [26]. After conversion to polar coordinates, his angular-momentum and boost operators are

$$
\underline{\mathbf{J}}_{\mathrm{Sh}}=-i \mathbf{p} \times \nabla+(\hat{\boldsymbol{\theta}} \cot \theta+\hat{\mathbf{p}}) \underline{S_{3}}
$$

and

$$
\underline{\mathbf{K}}_{\mathrm{Sh}}=i p \nabla+\hat{\boldsymbol{\phi}} \cot \theta \underline{S_{3}} .
$$

The Białynicki-Birulas 27,28 derived results equivalent to Eqs.(12) and (13) by considering the Maxwellian momentum and angular-momentum tensors. They also found [28] a unitary matrix $W_{1}$ that relates operators in the Foldy and Shirokov representations:

$$
\underline{O}_{\mathrm{F}}=\underline{W}_{1} \underline{O}_{\mathrm{Sh}}^{(1)} \underline{W}_{1}^{-1}
$$

We can express their $\underline{W_{1}}$ as the product of a transformation $\underline{T}$ from a Cartesian to an angular-momentum basis and another rotation

$$
\underline{D}(\phi, \theta, 0)=\exp \left(-i \underline{S_{3}} \phi\right) \exp \left(-i \underline{S_{2}} \theta\right)
$$

that takes $\mathbf{e}_{3}$ into $\hat{\mathbf{p}}: \underline{W}_{1}=\underline{D}(\phi, \theta, 0) \underline{T}=\underline{T} \underline{D}^{(1)}(\phi, \theta, 0)$. The unitary transformation

$$
\underline{T}=\frac{1}{\sqrt{2}}\left(\begin{array}{ccc}
1 & 0 & 1 \\
i & 0 & -i \\
0 & \sqrt{2} & 0
\end{array}\right)
$$


is required only because different bases for the momentum-space vectors have been assumed in the two representations. Specifically in the transformation (14), the Foldy operator $\underline{O}_{\mathrm{F}}$ uses a Cartesian basis, in which the unit vectors $\mathbf{e}_{1}, \mathbf{e}_{2}, \mathbf{e}_{3}$ are represented by column vectors with elements $\left(\mathbf{e}_{j}\right)_{k}=\delta_{j k}$, whereas $\underline{O}_{\mathrm{Sh}}^{(1)}$ uses an angular-momentum basis in which the complex unit vectors $\mathbf{e}_{\kappa}, \kappa= \pm 1,0$, are column matrices with elements $\left(\mathbf{e}_{\kappa}\right)_{k}=\delta_{\kappa, k-2}$. They are given in the Cartesian basis by the columns of $\underline{T}$ (16). The spin matrices are different in the two bases. In the Cartesian basis, the matrices $S_{j}$ have elements $\left(S_{j}\right)_{k l}=-i \varepsilon_{j k l}$ whereas in the spin-1 angular-momentum basis the corresponding matrices are $\left(S_{3}^{(1)}\right)_{k l}=(2-k) \delta_{k l}$, and $\left(S_{1}^{(1)} \pm i S_{2}^{(1)}\right)_{k l}=\sqrt{2} \delta_{k, l \pm 1}$. The body rotation matrices, expressed in terms of the Euler angles $\phi, \theta, \chi$ by

$$
\underline{D}(\phi, \theta, \chi)=\exp \left(-i \underline{S_{3}} \phi\right) \exp \left(-i \underline{S_{2}} \theta\right) \exp \left(-i \underline{S_{3}} \chi\right)
$$

have correspondingly different elements in the two bases. Explicit values in the Cartesian basis are given in the following section and in Appendix A. Elements of $\underline{D}^{(1)}=\underline{T}^{-1} \underline{D} \underline{T}$ in the angular-momentum basis have the standard form $D_{\kappa \kappa^{\prime}}^{(1)}=\exp \left(-i \kappa \phi-i \kappa^{\prime} \chi\right) d_{\kappa \kappa^{\prime}}^{(1)}(\theta)$, where the real matrix $\underline{d}^{(1)}$ is 29

$$
\underline{d}^{(1)}(\theta)=\frac{1}{2}\left(\begin{array}{ccc}
\cos \theta+1 & \sqrt{2} \sin \theta & \cos \theta-1 \\
-\sqrt{2} \sin \theta & 2 \cos \theta & -\sqrt{2} \sin \theta \\
\cos \theta-1 & \sqrt{2} \sin \theta & \cos \theta+1
\end{array}\right) .
$$

We will generally assume that the different representations both use the Cartesian basis or both use an angular-momentum basis for their momentum-space vectors, as is actually implicit in relation (8) between the Foldy and Lomont-Moses representations. We can then omit the factor $\underline{T}$ and replace the transformation (14) by

$$
\underline{O}_{\mathrm{F}}=\underline{D}(\phi, \theta, 0) \underline{O}_{\mathrm{Sh}} \underline{D}^{-1}(\phi, \theta, 0) .
$$

The helicity representations of Lomont and Moses and of Shirokov are both seen to be unitarily equivalent to the standard Foldy representation, and the unitary transformations for both are rotations that rotate $\underline{S_{3}}$ into $\underline{\mathbf{S}} \cdot \hat{\mathbf{p}}$. It seems surprising that the two helicity 
representations appear so different. As Chakrabarti [24 points out, the Shirokov form of the operators $\mathbf{J}$ and $\mathbf{K}$ are of a singular nature that makes them appear unsatisfactory for many uses. However, the Lomont and Moses form (10,11) also has first-order singularities at $\theta=\pi$, they are just better disguised. The differences in the unitary transformations (8) and (19) is clearer if $\underline{U}$ is expressed in terms of Euler angles. Since

$$
\exp \left(-i \underline{S_{3}} \phi\right) \underline{S_{2}} \exp \left(i \underline{S_{3}} \phi\right)=\underline{S_{2}} \cos \phi-\underline{S_{1}} \sin \phi=\underline{\mathbf{S}} \cdot \hat{\phi}
$$

then from the Euler-angle form (17) we can equate

$$
\underline{U}=\underline{D}(\phi, \theta,-\phi),
$$

that is, the unitary transformation $\underline{U}$ that rotates $\underline{S_{3}}$ directly to $\underline{\mathbf{S}} \cdot \hat{\mathbf{p}}$ is just the Euler-angle rotation $\underline{D}(\phi, \theta, 0)$ required for the Shirokov form preceded by an axial rotation through the angle $-\phi$. It is important to note that the transformations involved are spin rotations and therefore transform only spin components and the vector components of the photon states. We explore a generalization of such rotations further in the next section.

While the position operators are not explicitly required to complete the Poincaré algebra, they are implicit in the angular momentum and boost operators since, apart from internal degrees of freedom, it is the components of $\mathbf{r} \times \mathbf{p}$ and $\frac{1}{2}(p \mathbf{r}+\mathbf{r} p)$ that generate rotations and boosts, respectively. One set of commonly accepted photon position operators consists of components of the Pryce operator [22,18],

$$
\underline{\mathbf{r}}_{\mathrm{P}}=i p^{\alpha} \nabla p^{-\alpha}+\frac{\mathbf{p} \times \underline{\mathbf{S}}}{p^{2}}
$$

The parameter $\alpha$ in the expression

$$
p^{\alpha} \nabla p^{-\alpha}=\nabla+\frac{\alpha}{p}[p, \nabla]=\nabla-\frac{\alpha \mathbf{p}}{p^{2}}
$$

depends on the integration weight used in the definition of the scalar product. Pryce used the parameter value $\alpha=1 / 2$, which is appropriate when the Hilbert-space states are electromagnetic fields, and this value was implicitly assumed in Eqs.(17), (111), and (13). However, 
one needs $\alpha=-\frac{1}{2}$ when the vector potential [30] is used for the photon states. Alternatively, a definition of scalar product requiring $\alpha=0$ can be used 18, 3. In the following, we leave $\alpha$ unspecified.

The Pryce position operator [22] $\underline{\mathbf{r}}_{\mathrm{P}}$ is based on expressions in terms of a classical energymomentum tensor of a noncovariant definition of the center of mass given by Fokker [31]. The components of $\underline{\mathbf{r}}_{\mathrm{P}}$ are $3 \times 3$ matrices that do not commute with $\underline{S_{j}}$. When expressed in terms of $\underline{\mathbf{r}}_{\mathrm{P}}$, the rotation and boost generators of Foldy [Eqs. (2) and ([)] are partitioned differently into orbital and spin parts

$$
\begin{aligned}
\underline{\mathbf{J}} & =-\mathbf{p} \times \underline{\mathbf{r}}_{\mathrm{P}}+\hat{\mathbf{p}} \hat{\mathbf{p}} \cdot \mathbf{S} \\
\underline{\mathbf{K}} & =\frac{1}{2}\left(\underline{\mathbf{r}}_{\mathrm{P}} p+p \underline{\mathbf{r}}_{\mathrm{P}}\right) .
\end{aligned}
$$

While components of $\underline{\mathbf{r}}_{\mathrm{P}}$ do satisfy the canonical commutation relations with $\mathbf{p}$, they do not commute with each other [32, 33]. Instead, they have a nonvanishing commutator that is analogous to the field of a Dirac monopole (but in momentum space) whose "charge" is given by the helicity operator $\hat{\mathbf{p}} \cdot \underline{\mathbf{S}}[18]$.

$$
\left[\underline{r}_{\mathrm{P} j}, \underline{r}_{\mathrm{P} k}\right]=-i \varepsilon_{j k l} \frac{p_{l}}{p^{3}} \hat{\mathbf{p}} \cdot \underline{\mathbf{S}} .
$$

As discussed in the next section, such monopole terms occur frequently in commutators with gauge potentials.

\section{PHOTON POSITION OPERATORS WITH COMMUTING COMPONENTS}

In I, a modification of the Pryce position operator was constructed to have commuting components. In this section we summarize the results of I, emphasizing how the new position operator can be expressed as a spin rotation of the weight-modified position operator for massive particles. We then extend the construction to an arbitrary functional dependence $\chi_{\mathbf{p}}(\theta, \phi)$ among the three Euler angles $\phi, \theta, \chi$.

The position operator

$$
\underline{\mathbf{r}}^{(0)}=i p^{\alpha} \nabla p^{-\alpha}+\frac{\mathbf{p} \times \underline{\mathbf{S}}}{p^{2}}-\mathbf{a}^{(0)} \hat{\mathbf{p}} \cdot \underline{\mathbf{S}},
$$


with $\mathbf{a}^{(0)}=\hat{\boldsymbol{\phi}} p^{-1} \cot \theta$, was constructed in Ref. [19] by requiring that the components of $\underline{\mathbf{r}}^{(0)}$ have transverse eigenvectors in the directions $\hat{\boldsymbol{\theta}}$ and $\hat{\boldsymbol{\phi}}$. The operator $\underline{\mathbf{r}}^{(0)}$ can be expressed as a spin rotation of $i p^{\alpha} \nabla p^{-\alpha}$ :

$$
\underline{\mathbf{r}}^{(0)}=i \underline{D}(\phi, \theta, 0) p^{\alpha} \nabla p^{-\alpha} \underline{D}^{-1}(\phi, \theta, 0) .
$$

Since this has the same form as (19), it is equivalent to using $i p^{\alpha} \nabla p^{-\alpha}$ as the corresponding position operator in the helicity representation. The construction (28) may be understood by analyzing the problems of the Pryce position operator (22). The reason components of $r_{\mathrm{P}}$ do not commute is that the photon spin and its momentum are inextricably coupled through the restriction on allowed spin states, and in particular, the components of the momentumspace wave function depend on momentum direction. Consequently, the action of the usual momentum-space position operator for massive particles, $i \nabla$, is no longer restricted to the argument of the wave function but also acts on its components. In expression (28), on the other hand, the spin rotation $D^{-1}$ rotates vector components of the state function from the lab to the reference frame, where $\hat{\mathbf{p}}=\mathbf{e}_{3}$, so that the operator $i \nabla$ can induce translations in its arguments without mixing its components. Finally, $D$ rotates the translated vector back to the lab frame. This is a straightforward way to restore the usual translational role of $i \nabla$, and it is required to ensure that helicity eigenstates are not mixed by infinitesimal momentum-space translations generated by $\underline{\mathbf{r}}^{(0)}$ away from the origin $p=0$. This is an important property that follows from the invariance of the helicity subspaces under the Poincaré group and has been confirmed, for example, in experiments demonstrating Berry's phase [34] for photons in curved optical fibers [35].

The term $\mathbf{a}^{(0)}=\hat{\boldsymbol{\phi}} p^{-1} \cot \theta$ multiplying the helicity operator $\hat{\mathbf{p}} \cdot \underline{\mathbf{S}}$ in the new position operator (27) is singular in the limits $\theta \rightarrow 0, \pi$, that is, as $\mathbf{p}$ approaches the $\pm \mathbf{e}_{3}$ axes, and has the momentum-space form of the electromagnetic vector potential of a pair of Dirac monopole strings in coordinate space. The relationship is discussed in more detail at the end of Section V. It seems curious for the position operator to depend on the choice of coordinates in the laboratory. The singularity together with the dependence on laboratory 
coordinates may have deterred other authors from including such a term. Nevertheless, we show below that some form of this term is required to give the correct phase changes of the photon state under rotations. Laboratory coordinates enter the formulation of $\underline{\mathbf{r}}^{(0)}$ in the spin rotation $\underline{D}$ from $\mathbf{e}_{3}$ to $\hat{\mathbf{p}}$ and in the implicit dependence of $\hat{\mathbf{p}}$ on its polar angles $\theta, \phi$. The singular term arises from the differentiation of this rotation and from the pathdependent value of $\phi$. It is consistent with the relatively large changes in $\phi$ that can result from infinitesimal changes in $\mathbf{p}$ at the string. In this section we explore a generalization of the rotation used in $\underline{\mathbf{r}}^{(0)}$ and show that the breakdown of the expected symmetry is related to the selection of a specific "gauge" for $\underline{\mathbf{r}}$.

The spin rotation that plays a central role in this paper can be written in Cartesian form as the real unitary matrix of elements

$$
D_{j k} \equiv \mathbf{e}_{j} \cdot \mathbf{e}_{\mathbf{p} k}
$$

that relate vector components in the orthonormal lab basis $\left\{\mathbf{e}_{1}, \mathbf{e}_{2}, \mathbf{e}_{3}\right\}$ to those in the photonmomentum basis $\left\{\mathbf{e}_{\mathbf{p} 1}, \mathbf{e}_{\mathbf{p} 2}, \mathbf{e}_{\mathbf{p} 3}\right\}$, where $\mathbf{e}_{\mathbf{p} 3} \equiv \hat{\mathbf{p}}$. Thus, for any vector $\mathbf{V}, V_{j}=\mathbf{V} \cdot \mathbf{e}_{j}=$ $D_{j k} \mathbf{V} \cdot \mathbf{e}_{\mathbf{p} k} \equiv D_{j k} V_{\mathbf{p} k}$. This gives the action of the matrix $\underline{D}$ as a passive transformation ( $\mathbf{V}$ is fixed, the "observer" basis changes). However, only the relative orientation of the vector to the observer basis enters the formalism, and in particular, $\mathbf{e}_{j} \cdot \mathbf{e}_{k}=\delta_{j k}=\mathbf{e}_{\mathbf{p} j} \cdot \mathbf{e}_{\mathbf{p} k}$. As a result, an active interpretation of the action of $\underline{D}$ (observer fixed, $\mathbf{V}$ changes) is also possible and often more natural. As an active transformation, $\underline{D}$ rotates each of the vectors $\underline{\mathbf{e}_{1}}, \underline{\mathbf{e}_{2}}, \underline{\mathbf{e}_{3}}$, of the lab frame into the corresponding vector $\underline{\mathbf{e}_{\mathbf{p} 1}}, \underline{\mathbf{e}_{\mathbf{p} 2}}, \underline{\mathbf{e}_{\mathbf{p} 3}}$, of the photon $\mathbf{p}$-frame, with both vectors expressed in the lab basis: $\underline{\mathbf{e}_{\mathbf{p} j}}=\underline{D} \underline{\mathbf{e}_{j}}$. The mathematical consistency of the different interpretations is readily confirmed by writing out the column matrix representations of the vectors. Further details are given in Appendix B.

The body rotation $\underline{D}$ generalizes the polar rotation used in I. One can express $\underline{D}$ in terms of spin matrices and the Euler angles $\phi, \theta$ and $\chi$ as in Eq. (17), and it is given explicitly in Eq. (B4). The treatment in I corresponds to the choice $\chi=0$, in which $\mathbf{e}_{\mathbf{p} 1}=\hat{\boldsymbol{\theta}}, \mathbf{e}_{\mathbf{p} 2}=\hat{\boldsymbol{\phi}}$, and $\mathbf{e}_{\mathbf{p} 3}=\hat{\mathbf{p}}$. For more general $\chi$ 


$$
\begin{aligned}
& \mathbf{e}_{\mathbf{p} 1}=\hat{\boldsymbol{\theta}} \cos \chi+\hat{\boldsymbol{\phi}} \sin \chi \\
& \mathbf{e}_{\mathbf{p} 2}=\hat{\boldsymbol{\phi}} \cos \chi-\hat{\boldsymbol{\theta}} \sin \chi
\end{aligned}
$$

The angle $\chi$ represents an axial rotation about $\hat{\mathbf{p}}$ and is associated below with the phase of a photon with definite helicity. As seen by inspection of Eqs. (30.31), the transverse unit vectors $\mathbf{e}_{\mathbf{p} 1}, \mathbf{e}_{\mathbf{p} 2}$, are just the polar unit vectors $\hat{\boldsymbol{\theta}}, \hat{\boldsymbol{\phi}}$, rotated about $\hat{\mathbf{p}}$ through the angle $\chi$, so that use of the Euler angle $\chi$ allows an arbitrary orientation of the two directions $\mathbf{e}_{\mathbf{p} 1}, \mathbf{e}_{\mathbf{p} 2}$, in the transverse plane. The unit vectors describing states of definite helicity $\kappa$,

$$
\begin{aligned}
\mathbf{e}_{\mathbf{p} \kappa} & \equiv \frac{1}{\sqrt{2}}\left(\mathbf{e}_{\mathbf{p} 1}+i \kappa \mathbf{e}_{\mathbf{p} 2}\right) \\
& =e^{-i \kappa \chi} \mathbf{e}_{\mathbf{p} \kappa}^{(0)},
\end{aligned}
$$

are just phase shifted relative to the helicity states $\mathbf{e}_{\mathbf{p} \kappa}^{(0)}=(\hat{\boldsymbol{\theta}}+i \kappa \hat{\boldsymbol{\phi}}) / \sqrt{2}$ with $\chi=0$. Under the general rotation (17), the spin matrices themselves are transformed as

$$
\underline{S_{\mathbf{p} j}}=\underline{D} \underline{S}_{j} \underline{D}^{-1}=\underline{S_{k}} D_{k j}
$$

where in particular $\underline{S_{\mathbf{p} 3}}=\underline{\mathbf{S}} \cdot \hat{\mathbf{p}}$. Consequently, we can also express $\underline{D}$ in terms of rotations about the $\mathbf{p}$-frame axes:

$$
\begin{aligned}
\underline{D} & =\underline{D} \underline{D} \underline{D}^{-1} \\
& =e^{-i \underline{\underline{D}} \underline{S_{3}} \underline{D}^{-1} \phi} e^{-i \underline{\underline{D}} \underline{S_{2}} \underline{\underline{D}} \underline{1}^{-1} \theta} e^{-i \underline{\underline{D}} \underline{S_{3}} \underline{\underline{D}}^{-1} \chi} \\
& =\exp \left(-i \underline{S_{\mathbf{p} 3}} \phi\right) \exp \left(-i \underline{S_{\mathbf{p} 2} \theta}\right) \exp \left(-i \underline{S_{\mathbf{p} 3}} \chi\right) .
\end{aligned}
$$

Alternatively, we can make the axial rotation the last of the Euler-angle rotations by putting $\underline{D}_{0} \equiv \exp \left(-i \underline{S_{3}} \phi\right) \exp \left(-i \underline{S_{2}} \theta\right)$ and noting

$$
\begin{aligned}
\underline{D} & =\underline{D}_{0} \exp \left(-i \underline{S}_{3} \chi\right) \underline{D}_{0}^{-1} \underline{D}_{0} \\
& =\exp \left(-i \underline{D} \underline{S_{3}} \underline{D}^{-1} \chi\right) \underline{D}_{0} \\
& =\exp (-i \underline{\mathbf{S}} \cdot \hat{\mathbf{p}} \chi) \exp \left(-i \underline{S_{3}} \phi\right) \exp \left(-i \underline{S_{2}} \theta\right) .
\end{aligned}
$$

The position operator that generalizes Eq. (27) is the $3 \times 3$ matrix 


$$
\begin{aligned}
\underline{\mathbf{r}} & =\underline{D}\left(i p^{\alpha} \nabla p^{-\alpha}\right) \underline{D}^{-1} \\
& =i p^{\alpha} \nabla p^{-\alpha}-\underline{\mathbf{A}}
\end{aligned}
$$

where

$$
\begin{aligned}
\underline{\mathbf{A}} & =-i \underline{D}\left(\nabla \underline{D}^{-1}\right)=i(\nabla \underline{D}) \underline{D}^{-1} \\
& =\frac{\underline{\mathbf{S}} \times \hat{\mathbf{p}}}{p}+\mathbf{a} \hat{\mathbf{p}} \cdot \underline{\mathbf{S}}
\end{aligned}
$$

and $\mathbf{a}$ is a vector with dimensions of length. If $\chi$ is independent of $\theta, \phi$, then the axial rotation elements commute with $\nabla$ and $\underline{\mathbf{r}}$ is given by $\underline{\mathbf{r}}^{(0)}$ as found in I. However, the momentum $\mathbf{p}$ is invariant under the axial rotation by $\chi$, and we can choose a functional dependence $\chi=\chi_{\mathbf{p}}(\theta, \phi)$. Then the matrix $\underline{D}$ is a function of the two parameters $\theta, \phi$, and direct calculation of the gradient of the rotation matrix gives

$$
\mathbf{a}=\mathbf{a}^{(0)}+\nabla \chi_{\mathbf{p}}
$$

with

$$
\mathbf{a}^{(0)}=\hat{\boldsymbol{\phi}} \frac{\cot \theta}{p}=\frac{\mathbf{e}_{3} \times \mathbf{p} \hat{\mathbf{p}} \cdot \mathbf{e}_{3}}{\left(\mathbf{e}_{3} \times \mathbf{p}\right)^{2}}
$$

When operating on a subspace of definite helicity $\kappa= \pm 1, \underline{\mathbf{r}}$ is related to the Pryce operator $\underline{\mathbf{r}}_{\mathrm{P}}$ of form (22) by a transverse displacement whose sign depends on $\kappa$ :

$$
\underline{\mathbf{r}}=\underline{\mathbf{r}}_{\mathrm{P}}-\kappa \mathbf{a} .
$$

This clearly manifests the basic coupling between the helicity (and therefore the spin) and the position. However, as we see in the following section, the eigenvalues of $\underline{\mathbf{r}}$ do not depend explicitly on $\kappa$.

The matrix $\underline{\mathbf{A}}$, with transverse vectors as its matrix elements, has a simple interpretation. Writing

$$
\nabla \underline{D}=-i \underline{\mathrm{A}} \underline{D}
$$

and making the dependence of the rotation matrix $\underline{D}$ on $\mathbf{p}$ explicit, we find 


$$
\begin{aligned}
\underline{D}(\mathbf{p}+d \mathbf{p}) & =\underline{D}(\mathbf{p})+d \mathbf{p} \cdot \nabla \underline{D}(\mathbf{p}) \\
& =(1-i d \mathbf{p} \cdot \underline{\mathbf{A}}) \underline{D}(\mathbf{p})
\end{aligned}
$$

Thus, $\underline{\mathbf{A}} \cdot d \mathbf{p}$ generates the infinitesimal rotation induced when $\mathbf{p}$ is incremented by $d \mathbf{p}$.

The possibility of choosing different functions $\chi_{\mathbf{p}}(\theta, \phi)$ dependent on the position $\theta, \phi$ in parameter space expresses the invariance of $\mathbf{p}$ under axial rotations as a local symmetry and is analogous to gauging a and hence $\underline{\mathbf{r}}$. The gauge group of axial rotations is $U(1)$, and $\mathbf{a}$ is thus analogous to the electromagnetic vector potential. The scalar gauging function $\chi_{\mathbf{p}}$ is a concrete example of Berry's gauge [34 that sets the local phase of an eigenstate in parameter space. It also appears closely related to the function $G_{0}$ introduced by Staruszkiewicz in his earlier study [36] of parallel transport of photons. For all choices of single-valued differentiable functions $\chi_{\mathbf{p}}$, the gauge field

$$
\nabla \times \mathbf{a}=-\frac{\hat{\mathbf{p}}}{p^{2}}, \quad \sin \theta \neq 0,
$$

has the same monopole form. It follows that $\mathbf{a}$ itself cannot be replaced by a gradient term. As we discuss below (see especially Section $V$ ) however, $\chi_{\mathbf{p}}$ is generally not single valued and can affect stringlike singularities in the field (50). The "abelian vector potential" $\mathbf{a}$ is just part of a nonabelian operator $\underline{\mathbf{A}}=\underline{\mathbf{S}} \times \hat{\mathbf{p}} / p+\mathbf{a} \hat{\mathbf{p}} \cdot \underline{\mathbf{S}}$.

Berry [34] has found analogous monopole fields (50) both for the case of a pair of adiabatic states that are degenerate at an isolated point in parameter space and for spin- $S$ charges in a magnetic field that are degenerate where the field vanishes. Our derivation shows that such fields arise more generally in calculations of topological phase, even when there is no isolated point of degeneracy. Indeed, monopole terms of the form $\hat{\mathbf{p}} / p^{2}$ frequently arise in the commutators involving gauge potentials associated with massless representations of the Poincaré group [18,28,32,33,37 40]. In our case, direct calculation gives

$$
\begin{aligned}
{\left[\underline{A}_{j}, \underline{A}_{k}\right] } & =i \varepsilon_{j k l}\left(\nabla_{\mathbf{p}} \times \underline{\mathbf{A}}\right) \cdot \mathbf{e}_{l} \\
& =i \varepsilon_{j k l}\left(\hat{\mathbf{p}} \cdot \underline{\mathbf{S}} \frac{\mathbf{a} \times \mathbf{p}+\hat{\mathbf{p}}}{p^{2}}+\frac{\underline{\mathbf{S}} \times \mathbf{a}}{p}\right) \cdot \mathbf{e}_{l},
\end{aligned}
$$


which includes the monopole term $\hat{\mathbf{p}} / p^{2}$ multiplied by the helicity operator $\hat{\mathbf{p}} \cdot \underline{\mathbf{S}}$. Similar terms also arise from the distinct "covariant derivatives" $\mathfrak{D}^{\prime}=\nabla-i \mathbf{p} \times \mathbf{S} / p^{2}$ and $\mathfrak{D}^{\prime \prime}=\nabla-$ $i \kappa \mathbf{a}$ introduced for photon operators by Pati [40] and the Białynicki-Birulas [28], respectively. The common appearance of monopole terms and their associated string singularity (see Section V) is a direct consequence of the topology of momentum space [36,41] (see also Appendix A). Here, the monopole "field" (50) turns out to be just what is required to cancel the singularity in the commutator (26) of the Pryce position operators. Furthermore, the relation $(\nabla+i \underline{\mathbf{A}}) \underline{D}=0$ implies that $\mathfrak{D}=\nabla+i \underline{\mathbf{A}}$ is the appropriate "covariant derivative" in our formulation.

There is a wide range of possible gauge choices since $\chi_{\mathbf{p}}(\theta, \phi)$ can in principle be any differentiable function. However, three choices of $\chi_{\mathbf{p}}$ are of particular interest: If $\chi_{\mathbf{p}}$ is the zero function, $\chi_{\mathbf{p}}^{(0)}=0$, then the transverse directions are the usual spherical polar unit vectors, $\mathbf{a}$ is just $\mathbf{a}^{(0)}(45)$, and $\underline{\mathbf{r}}$ is given by Eq.(27). If $\chi_{\mathbf{p}}$ is $\chi_{\mathbf{p}}^{(1)}=-\phi$, then as noted in Section II, $\underline{D}$ is the Chakrabarti [24] transformation $\underline{U}$ (9), and a takes the form

$$
\mathbf{a}^{(1)}=\frac{\cos \theta-1}{p \sin \theta} \hat{\boldsymbol{\phi}}=\frac{\hat{\mathbf{p}} \times \mathbf{e}_{3}}{p+\mathbf{p} \cdot \mathbf{e}_{3}}
$$

in Eq.(41). We note that this choice eliminates the singularity in a at $\theta=0$, but doubles the strength of the one at $\theta=\pi$ (see also Section V). If instead $\chi_{\mathbf{p}}$ is $\chi_{\mathbf{p}}^{(2)}=-\phi \cos \theta$, then $\mathbf{a}$ is given by

$$
\mathbf{a}^{(2)}=\frac{\hat{\boldsymbol{\theta}}}{p} \phi \sin \theta .
$$

In this case, both string singularities are removed, but a is now path dependent, since the value of $\phi$ depends on how many times the path wraps around the $\mathbf{e}_{3}$ axis. Consequently, $\mathbf{a}^{(2)}$ is "nonintegrable" 38]. By subtracting another path-dependent term, it is also possible to reintroduce a string singularity about a different axis. 


\section{POINCARÉ GENERATORS AND COMMUTATION RELATIONS}

Here we examine the position operator $\underline{\mathbf{r}}$ in the context of the Poincaré algebra and write explicit momentum-space expressions for the localized bases. To see the relationship of our position operator $\underline{\mathbf{r}}$ and the Poincaré operators in the existing literature, note that translation generators $\mathbf{p}$ and $H=p c$ are unchanged, and the Foldy $\underline{\mathbf{J}}$ and $\underline{\mathbf{K}}$ operators can be written in terms of an orbital angular momentum operator

$$
\underline{\mathbf{L}}^{(r)} \equiv \underline{\mathbf{r}} \times \mathbf{p}
$$

corresponding to our position operator (41), and an effective spin operator, which has a transverse contribution in addition to the helicity term that is frequently assumed to be the total spin contribution [32,33,42],

$$
\underline{\mathbf{S}}^{(r)} \equiv(\mathbf{a} \times \mathbf{p}+\hat{\mathbf{p}}) \hat{\mathbf{p}} \cdot \underline{\mathbf{S}}
$$

We obtain

$$
\underline{\mathbf{J}}=\underline{\mathbf{L}}^{(r)}+\underline{\mathbf{S}}^{(r)}
$$

and

$$
\begin{aligned}
\underline{\mathbf{K}} & =\frac{1}{2}(p \underline{\mathbf{r}}+\underline{\mathbf{r}} p)+\hat{\mathbf{p}} \times \underline{\mathbf{S}}^{(r)} \\
& =\frac{1}{2}(p \underline{\mathbf{r}}+\underline{\mathbf{r}} p)+\mathbf{a} \mathbf{p} \cdot \underline{\mathbf{S}}
\end{aligned}
$$

as the Foldy operators (generalized to arbitrary $\alpha$ ) in terms of our position operator. With these expressions, transformation to one of the helicity forms is straightforward. By inverting Eqs.(40) and (34) the position operator becomes $\underline{D}^{-1} \underline{\mathbf{r}} \underline{D}=i p^{\alpha} \nabla p^{-\alpha}$, while $\underline{D}^{-1} \hat{\mathbf{p}} \cdot \underline{\mathbf{S}} \underline{D}=\underline{S_{3}}$. The general helicity representation is given by $\underline{O}_{\mathrm{h}}=\underline{D}^{-1} \underline{O D}$, which gives

$$
\begin{aligned}
\underline{\mathbf{J}}_{\mathrm{h}} & =\left(i p^{\alpha} \nabla p^{-\alpha}\right) \times \mathbf{p}+(\mathbf{a} \times \mathbf{p}+\hat{\mathbf{p}}) \underline{S_{3}} \\
\underline{\mathbf{K}}_{\mathrm{h}} & =\frac{i}{2} p^{\alpha}(p \nabla+\nabla p) p^{-\alpha}+\mathbf{a} p \underline{S_{3}}
\end{aligned}
$$


If $\chi_{\mathbf{p}}=0$, then $\mathbf{a}=\hat{\phi} \cot \theta / p$ and $\underline{\mathbf{J}}_{\mathrm{h}}$ and $\underline{\mathbf{K}}_{\mathrm{h}}$ reduce to the Shirokov operators given by Eqs.(12) and (13) generalized to arbitrary $\alpha$, while if $\chi_{\mathbf{p}}=-\phi$, $\mathbf{a}$ is given by $\hat{\boldsymbol{\phi}}(\cos \theta-1) /(p \sin \theta)=-\hat{\boldsymbol{\phi}} \sin \theta /(1+\cos \theta)$ and $\underline{\mathbf{J}}_{\mathrm{h}}$ and $\underline{\mathbf{K}}_{\mathrm{h}}$ reduce to the Lomont and Moses operators, Eqs.(10) and (11) with arbitrary $\alpha$. Thus both the Lomont and Moses and the Shirokov transformations are special cases of Euler angle rotations relative to the Foldy form. In any irreducible helicity representation the position operator is just $i p^{\alpha} \nabla p^{-\alpha}$ as it is for massive particles in the Foldy form, since the transverse directions are fixed in the reference frame where the photon momentum is parallel to $\mathbf{e}_{3}$. The helicity reference frame is the photon frame, while the Foldy frame is the lab frame.

The commutation relations satisfied by the generators of time and space translations, rotations and boosts, $H=p c, p_{i}, \underline{J_{j}}$ and $\underline{K_{j}}$, are the standard ones required by the Poincaré algebra: $\left[\underline{J_{j}}, \underline{J_{k}}\right]=i \epsilon_{j k l} \underline{J_{l}},\left[\underline{J_{j}}, \underline{K_{k}}\right]=i \epsilon_{j k l} \underline{K_{l}},\left[\underline{K_{j}}, \underline{K_{k}}\right]=-i \epsilon_{j k l} \underline{J_{l}},\left[\underline{J_{j}}, p_{k}\right]=i \epsilon_{j k l} p_{l}$, $\left[\underline{K_{j}}, p_{k}\right]=i \delta_{j k} H / c,\left[\underline{K_{j}}, H\right]=i c p_{j},\left[\underline{J_{j}}, H\right]=0,\left[p_{j}, H\right]=0$. Analogous commutation relations are valid if ${\underline{L_{j}}}^{(r)}$ is substituted for $\underline{J_{j}}$ and $\left(p \underline{r_{j}}+\underline{r_{j} p}\right) / 2$ for $\underline{K_{j}}$, since they are unitarily equivalent to the zero-spin case of the above. Commutation relations involving $\underline{r_{j}}$ in the lab frame can be most simply derived by performing a matrix rotation to the photon reference frame, in which $\underline{\mathbf{r}}$ is replaced by $i p^{\alpha} \nabla p^{-\alpha}$ and noting that components $p_{j}$ in the momentum representation commute with the rotation matrix $\underline{D}$. Thus $\left[\underline{r_{j}}, \underline{r_{k}}\right]=0$, $\left[\underline{r_{j}}, p_{k}\right]=i \delta_{j k},\left[\underline{r_{j}}, H\right]=i H p_{j} / p^{2},\left[\underline{L}_{j}^{(r)}, \underline{r_{k}}\right]=i \epsilon_{j k l} \underline{r_{l}},\left[\underline{r_{j}}, \underline{S_{\mathbf{p} k}}\right]=0$ and $\left[\underline{L}_{j}^{(r)}, \underline{S_{\mathbf{p} k}}\right]=0$. It follows from the last two relations that a photon can simultaneously have definite helicity and either a definite position or a well-defined spatial-component of orbital angular momentum. In other words, measurement of either the position or the orbital angular momentum of a photon state does not change its helicity. Furthermore, because

$$
\left[\underline{J_{k}}, \hat{\mathbf{p}} \cdot \mathbf{S}\right]=0,\left[\underline{K}_{k}, \hat{\mathbf{p}} \cdot \mathbf{S}\right]=0,\left[p_{k}, \hat{\mathbf{p}} \cdot \mathbf{S}\right]=0
$$

the helicity is an invariant of the Poincaré group; it is invariant under all rotations, boosts, and translations (as long as these avoid the origin $p=0$ ). Consequently, every representation of the Poincaré group for photons can be reduced to the direct sum of representations for the 
two helicities, and every irreducible representation will be carried by states of a single helicity. It may therefore be convenient to specify the operators for the separate invariant helicity subspaces. The momentum and energy operators are unchanged, the position operator is as given above in Eq. (46), and $\underline{\mathbf{J}}$ and $\underline{\mathbf{K}}$ take exactly their Pryce forms

$$
\underline{\mathbf{J}}=\underline{\mathbf{L}}^{(r)}+\underline{\mathbf{S}}^{(r)}=\underline{\mathbf{r}}_{\mathrm{P}} \times \mathbf{p}+\hat{\mathbf{p}} \kappa
$$

and

$$
\underline{\mathbf{K}}=\frac{1}{2}(p \underline{\mathbf{r}}+\underline{\mathbf{r}} p)+\mathbf{a} \kappa=\frac{1}{2}\left(p \underline{\mathbf{r}}_{\mathrm{P}}+\underline{\mathbf{r}}_{\mathrm{P}} p\right)
$$

with $\underline{\mathbf{r}}_{\mathrm{P}}=\underline{\mathbf{r}}+\kappa \mathbf{a}$.

It is important that our position operators obey the correct dynamical equations. In the Heisenberg picture, the dynamics are determined by the equation of motion, which from Eq. (40) is

$$
\frac{d \underline{\mathbf{r}}}{d t}=\frac{\partial \underline{\mathbf{r}}}{\partial t}+i[H, \underline{\mathbf{r}}]=i[c p, \underline{\mathbf{r}}]=c \hat{\mathbf{p}}
$$

Thus our theory predicts that the photon has a velocity $\mathbf{v}=c \hat{\mathbf{p}}$, as required. We also note that $\underline{\mathbf{r}}(41)$ is Hermitian and symmetric under time reversal. One may in addition expect $\underline{\mathbf{r}}$ to change sign under parity inversion, but this depends on the gauge potential. It is valid for $\underline{\mathbf{r}}^{(0)}$, but for other gauges we must generally replace the inversion in $\chi_{\mathbf{p}}$ by the gauge change $\chi_{\mathbf{p}} \rightarrow-\chi_{\mathbf{p}}$ in order to ensure the invariance of $\nabla \chi_{\mathbf{p}}$.

In general, the position operator rotates as a simple vector under $\underline{\mathbf{L}}^{(r)}$ since $\left[\underline{L}_{j}^{(r)}, \underline{r_{k}}\right]=$ $i \epsilon_{j k l} \underline{r_{l}}$, but not under $\underline{\mathbf{J}}$ :

$$
\left[\underline{J_{j}}, \underline{r_{k}}\right]=i \epsilon_{j k l} \underline{r_{l}}+\left[\underline{S}_{j}^{(r)}, \underline{r_{k}}\right]
$$

From $\left[\hat{\mathbf{p}} \cdot \underline{\mathbf{S}}, \underline{r_{j}}\right]=0$ and Eq. $(\underline{56})$, we thus find

$$
\begin{aligned}
{\left[\underline{J_{j}}, \underline{r_{k}}\right] } & =i \epsilon_{j k l} \underline{r_{l}}+\left[(\mathbf{a} \times \mathbf{p}+\hat{\mathbf{p}}) \cdot \mathbf{e}_{j}, \underline{r_{k}}\right] \kappa \\
& =i \epsilon_{j k l} \underline{r_{l}}-i \kappa\left\{\frac{\partial}{\partial p_{k}}(\mathbf{a} \times \mathbf{p}+\hat{\mathbf{p}}) \cdot \mathbf{e}_{j}\right\} .
\end{aligned}
$$


The extra term represents a deviation from the usual commutator (3), and is due to the coupling of the momentum and spin of a photon. Note that the deviation vanishes for rotations about $\hat{\mathbf{p}}$. It also vanishes in the special case of a rotation about $\mathbf{e}_{3}$ when $\chi_{\mathbf{p}}$ is independent of $\phi$.

For a photon with helicity $\kappa$ the momentum-space states localized at $\mathbf{r}$ are

$$
\underline{\Psi_{\mathbf{r}^{\prime}, \kappa}}(\mathbf{p})=N p^{\alpha} e^{-i \mathbf{r}^{\prime} \cdot \mathbf{p}} \underline{\mathbf{e}_{\mathbf{p} \kappa}},
$$

as may be verified by direct application of the operator $\mathbf{r}$ (40). In particular, the localized state at the origin,

$$
\underline{\Psi_{0, \kappa}}(\mathbf{p})=N p^{\alpha} \underline{\mathbf{e}_{\mathbf{p} \kappa}}
$$

gives

$$
\underline{\mathbf{L}}^{(r)} \underline{\mathbf{e}_{\mathbf{p} \kappa}}=-\mathbf{p} \times \underline{\mathbf{r}} \underline{\mathbf{e} \kappa}=0
$$

since $\underline{\Psi_{0, \kappa}}$ is an eigenvector of $\underline{\mathbf{r}}$ with eigenvalue 0 . The relation $\underline{\mathbf{r}} \underline{\mathbf{e}_{\mathbf{p} \kappa}}=0$ also follows directly from the transformations (40) and (B1). Using $\underline{\mathbf{J}}=\underline{\mathbf{L}}^{(r)}+\underline{\mathbf{S}}^{(r)}$ where $\underline{\mathbf{S}}^{(r)}$ is given by Eq.(56), we find the rotation by an infinitesimal angle $d \xi$ about the axis $\hat{\boldsymbol{\xi}}$ to be

$$
\begin{aligned}
\exp (-i \underline{\mathbf{J}} \cdot d \boldsymbol{\xi}) \underline{\mathbf{e}_{\mathbf{p} \kappa}} & =\exp \left(-i \underline{\mathbf{S}}^{(r)} \cdot d \boldsymbol{\xi}\right) \underline{\mathbf{e}_{\mathbf{p} \kappa}} \\
& =\exp (-i \kappa(\mathbf{a} \times \mathbf{p}+\hat{\mathbf{p}}) \cdot d \boldsymbol{\xi}) \underline{\mathbf{e}_{\mathbf{p} \kappa}}
\end{aligned}
$$

with $d \boldsymbol{\xi}=\hat{\boldsymbol{\xi}} d \xi$. Thus the rotated transverse basis vectors of helicity $\kappa$ satisfy

$$
\exp (-i \underline{\mathbf{J}} \cdot d \boldsymbol{\xi}) \underline{\mathbf{e}_{\mathbf{p} \kappa}}=\exp \left[-i \kappa\left(d \chi-d \chi_{\mathbf{p}}\right)\right] \underline{\mathbf{e}_{\mathbf{p} \kappa}}
$$

and are thus changed only by an infinitesimal phase shift

$$
-\kappa\left(d \chi-d \chi_{\mathbf{P}}\right)=-\kappa(\mathbf{a} \times \mathbf{p}+\hat{\mathbf{p}}) \cdot d \boldsymbol{\xi}
$$

Here, $d \chi_{\mathbf{p}}=d \mathbf{p} \cdot \nabla \chi_{\mathbf{p}}$ is the change in the function $\chi_{\mathbf{p}}(\theta, \phi)$ that results from the change $d \mathbf{p}$ in the photon momentum. (Note that $\mathbf{a}$ and $\underline{\mathbf{S}}^{(r)}$ depend on the orientation of $\hat{\mathbf{p}}$, and 
therefore ordered integral expressions of the Dyson type are required for finite rotations. See also the Appendix.)

Since $\mathbf{e}_{\mathbf{p} 0} \equiv \hat{\mathbf{p}}$ is a longitudinal basis vector corresponding to helicity $\kappa=0$, it follows from $\hat{\mathbf{p}} \cdot \underline{\mathbf{S}} \underline{\mathbf{e}_{\mathbf{p} 0}}=0$ that Eq. $(72)$ is also true of the longitudinal vector. Indeed, Eq. (72) may be considered an extension of the expected invariance of the radial vector field $\hat{\mathbf{p}}$ under rotations. Also, if $\hat{\boldsymbol{\xi}}=\boldsymbol{e}_{3}$, and $\chi_{\mathbf{p}}$ is constant, then it is readily shown that $d \chi-d \chi_{\mathbf{P}}=0$ so that this cylindrically symmetric transverse vector field is invariant under rotations about $\mathbf{e}_{3}$. Note further from Eq. (49) that with $d \mathbf{p}=d \boldsymbol{\xi} \times \mathbf{p}$, the infinitesimal rotation factor becomes

$$
\underline{D}(\mathbf{p}+d \mathbf{p}) \underline{D}^{-1}(\mathbf{p})=1-i \underline{\mathbf{A}} \cdot d \mathbf{p}=1-i\left(\mathbf{S}-\mathbf{S}^{(r)}\right) \cdot d \boldsymbol{\xi}
$$

The part $-i \mathbf{S} \cdot d \boldsymbol{\xi}$ gives the additional rotation whereas $i \mathbf{S}^{(r)} \cdot d \boldsymbol{\xi}$ corrects the axial rotation implied by the functional dependence $\chi_{\mathbf{p}}(\theta, \phi)$.

One can understand the extra term in the commutator of the position with the angular momentum by comparing Eqs.(67) and (73). Rotations generally change the "gauge potential" a in a way analogous to transformations of the electromagnetic potential $A$ under Lorentz transformations. The $\partial(\mathbf{a} \times \mathbf{p}+\hat{\mathbf{p}}) \cdot \mathbf{e}_{j} / \partial p_{i}$ term in Eq.[67) is required in order to give the axial spin rotation needed by the matrices associated with each component of $\underline{\mathbf{r}}$. Such axial rotations result in the phase change of eigenvectors during a rotation that maintains the functional dependence of $\chi_{\mathbf{p}}$ and is required in order to give the correct Berry's phase for photons (see the next section). The possible transformations are discussed in more detail in the Appendices, and Eqs.(73) and (67) are independently derived in Appendices B and $\mathrm{C}$ respectively.

\section{RELATION TO BERRY'S PHASE}

The phase angle $-\kappa\left(d \chi-d \chi_{\mathbf{p}}\right)$ is important and has measurable consequences. In particular, it can be integrated to give the total phase change of a state vector of helicity $\kappa$ 
when transported along a closed loop in parameter space. The result depends on the type of transport, but it will not depend on any single-valued functional choice of $\chi_{\mathbf{p}}$. However, it is natural to make $\chi_{\mathbf{p}}$ multi-valued and path-dependent (nonintegrable), and in that case the phase change will depend linearly on the change $\Delta \chi_{\mathbf{p}}$ around the closed loop, as we show explicitly below. If we use parallel transport, the angle $-\kappa\left(d \chi-d \chi_{\mathbf{p}}\right)$ is Berry's phase [34,43] $\gamma_{\kappa}$, a topological phase accumulated by the photon. This is consistent with a more general association of Berry's phase in quantum systems with geometrical angles. [44]

While Berry's derivation assumed adiabatic transport of energetically discrete states, his results can also be applied to degenerate helicity states in parallel transport 44,35]. Parallel transport on the spherical surface of constant radius $p$ is most easily realized by piecing the path together from many small segments of great circles and employing nonrotating transport along each segment. Each great-circle segment requires an axis of rotation that is perpendicular to $\mathbf{p}$ but generally changes as $\mathbf{p}$ moves. From Eqs. (73) and (72), the accumulated phase for great-circle segment $(\hat{\mathbf{p}} \cdot d \boldsymbol{\xi}=0)$ is

$$
\begin{aligned}
-\kappa\left(\Delta \chi-\Delta \chi_{\mathbf{p}}\right) & =-\kappa \oint \mathbf{a} \times \mathbf{p} \cdot d \boldsymbol{\xi} \\
& =-\kappa \oint \mathbf{a} \cdot \mathbf{p} \times d \boldsymbol{\xi} \\
& =\kappa \oint \mathbf{a} \cdot d \mathbf{p},
\end{aligned}
$$

where we noted $d \mathbf{p}=d \boldsymbol{\xi} \times \boldsymbol{p}$. The same result (75) can be obtained directly from Berry's derivation [34] of the geometric phase $\gamma_{\kappa}$. We can write his starting point in differential form as

$$
d \gamma_{\kappa}=i\left\langle u_{\kappa}(\mathbf{p}) \mid \nabla u_{\kappa}(\mathbf{p})\right\rangle \cdot d \mathbf{p}
$$

since the relevant parameter for the photon as it is guided in an optical fiber is its momentum $\mathbf{p}$. In our case, the eigenstate $u_{\kappa}(\mathbf{p})$ is proportional to the column vector

$$
\underline{\mathbf{e}_{\mathbf{p} \kappa}}=\underline{D} \underline{\mathbf{e}_{\kappa}}
$$

so that Berry's relation (76) reduces to 


$$
\begin{aligned}
d \gamma_{\kappa} & =i\left\langle\underline{\mathbf{e}}^{\dagger} \underline{D}^{\dagger}(\nabla \underline{D}) \underline{\mathbf{e}_{\kappa}}\right\rangle \cdot d \mathbf{p}=\left\langle\underline{\mathbf{e}_{\kappa}} \underline{D}^{\dagger} \underline{\mathbf{A}} \underline{D} \underline{\mathbf{e}_{\kappa}}\right\rangle \cdot d \mathbf{p} \\
& =\left\langle\underline{\mathbf{e}_{\mathbf{p} \kappa}} \underline{\mathbf{A}} \underline{\mathbf{e}_{\mathbf{p} \kappa}}\right\rangle \cdot d \mathbf{p}=\kappa \mathbf{a} \cdot d \mathbf{p}
\end{aligned}
$$

where the matrix sandwich indicated by angular brackets $\langle\cdots\rangle$ is a vector-valued $1 \times 1$ matrix, and we have noted that $\underline{\mathbf{S}} \times \hat{\mathbf{p}}$ has a vanishing diagonal in the $\underline{\mathbf{e}_{\mathbf{p} \kappa}}$ basis. In terms of the polar angles $\theta, \phi$, displacement on the spherical surface is given by $d \mathbf{p}=\hat{\boldsymbol{\phi}} p \sin \theta d \phi+\hat{\boldsymbol{\theta}} p d \theta$, and therefore with Eqs. (44, 45) for a, the line integral (75) gives

$$
\begin{aligned}
-\kappa\left(\Delta \chi-\Delta \chi_{\mathbf{p}}\right) & =\kappa\left(\oint \cos \theta d \phi+\oint \nabla \chi_{\mathbf{p}} \cdot d \mathbf{p}\right) \\
& =\kappa\left(\oint \cos \theta d \phi+\Delta \chi_{\mathbf{p}}\right) .
\end{aligned}
$$

The result depends on the function $\chi_{\mathbf{p}}$. We must choose a function that eliminates superfluous axial rotations. Recall that the basis vectors $\underline{\mathbf{e}_{\mathbf{p} \kappa}}$ are obtained from the reference frame by the rotation $\underline{D}\left(\phi, \theta, \chi_{\mathbf{p}}\right) \underline{\mathbf{e}}_{\kappa}$. To avoid unwanted axial rotations, we ensure that $\mathbf{p}$ is also parallel transported by this rotation. This requires the function choice

$$
\chi_{\mathbf{p}}(\theta, \phi)=\chi_{\mathbf{p}}^{(1)}(\theta, \phi)=-\phi
$$

to make $\underline{D}\left(\phi, \theta, \chi_{\mathbf{p}}\right)$ equivalent to the direct great-circle rotation from $\mathbf{e}_{3}$ to $\hat{\mathbf{p}}$. The phase shift is then

$$
\gamma_{\kappa}=-\kappa\left(\Delta \chi-\Delta \chi_{\mathbf{p}}^{(1)}\right)=\kappa(\oint \cos \theta d \phi-2 \pi)=-\kappa \Omega
$$

where $\Omega$ is the solid angle enclosed by the loop. This is Berry's geometrical phase 34 which has been confirmed in experiments on light in helically wound optical fibers [35]. The sign convention [34] is taken such that the dynamic phase of a stationary state (due to a factor $\exp (-i \omega t))$ decreases in time (see also Appendix D).

One often seeks to generalize the above result by an application of Stokes theorem. In our case

$$
\begin{aligned}
\oint \mathbf{a}^{(1)} \cdot d \mathbf{p} & =\int_{\Omega}\left(\nabla \times \mathbf{a}^{(1)}\right) \cdot \hat{\mathbf{p}} p^{2} d \Omega \\
& =-\int_{\Omega} \hat{\mathbf{p}} \cdot \frac{\hat{\mathbf{p}}}{p^{2}} p^{2} d \Omega=-\Omega
\end{aligned}
$$


where $\Omega$ is the solid angle of the integrated area. The result should be valid as long as the integrated area and its boundary avoid singularities and branch cuts. Since it is the curl of $\mathbf{a}$ that appears in the surface integration (83), gauge transformations $\mathbf{a} \rightarrow \mathbf{a}+\nabla \chi_{\mathbf{p}}$ will not change the result if $\chi_{\mathbf{p}}$ is a single-valued function of $\mathbf{p}$. This is consistent with the line integral (80) since for any single-valued $\chi_{\mathbf{p}}$, the difference $\Delta \chi_{\mathbf{p}}$ vanishes over a closed loop.

If, as in the optical-fiber experiments [35], $\mathbf{p}$ describes a circular path that makes a fixed angle $\theta$ with any fixed direction, then $\Omega=2 \pi(1-\cos \theta)$ so that the change in phase angle is

$$
\gamma_{\kappa}=\kappa \oint d \mathbf{p} \cdot \mathbf{a}^{(1)}=2 \pi \kappa(\cos \theta-1) .
$$

However, the derivation also apparently works if $\mathbf{a}^{(1)}$ is replaced by $\mathbf{a}^{(0)}$, even though these results should differ by $\kappa \Delta \chi_{\mathbf{p}}^{(1)}=-2 \pi \kappa$ for a closed loop around $\mathbf{e}_{3}$. While such a difference is not observable, we can trace its origin to the nonintegrable nature of $\chi_{\mathbf{P}}^{(1)}$ and the associated string singularity through the integrated area of the surface integral (B3). The argument can in fact be turned around to imply that because the difference cannot be observable, the helicity must be quantized.

Since the string contribution is frequently ignored, it may be useful to elaborate its role. Recall that Dirac's magnetic monopole contains a string to bring the magnetic flux to the monopole inside a solenoid with a vanishing diameter. Although strings are not evident in our expression (50) of $\nabla \times \mathbf{a}$, their presence is implied by the expression (44) for $\mathbf{a}^{(0)}$. The physics is clearer if we write $\mathbf{a}^{(0)}$ as the limit of a nonsingular function:

$$
\mathbf{a}^{(0)}=\hat{\phi} \lim _{p_{0} \rightarrow 0} \frac{p_{\perp} \cos \theta}{p_{\perp}^{2}+p_{0}^{2}}
$$

where $p_{\perp} \equiv p \sin \theta$ is the momentum-space distance from the $\mathbf{e}_{3}$ axis and $p_{0}$ represents the approximate diameter of the solenoid. The "magnetic field" corresponding to the "vector potential" is

$$
\nabla \times \mathbf{a}^{(0)}=\lim _{p_{0} \rightarrow 0}\left[-\hat{\mathbf{p}} \frac{\sin ^{2} \theta}{p_{\perp}^{2}+p_{0}^{2}}+\mathbf{e}_{3} \frac{2 p_{0}^{2} \cos \theta}{\left(p_{\perp}^{2}+p_{0}^{2}\right)^{2}}\right]
$$


The first term becomes the monopole field in the limit, and the second term represents the two axial strings. It vanishes everywhere except on the axis and can be expressed as a two-dimensional Dirac delta function, giving

$$
\nabla \times \mathbf{a}^{(0)}=-\frac{\hat{\mathbf{p}}}{p^{2}}+2 \pi \mathbf{e}_{3} \cos \theta \delta^{(2)}\left(p_{\perp}\right)
$$

where we can also write $\delta^{(2)}\left(p_{\perp}\right)=\delta\left(p_{1}\right) \delta\left(p_{2}\right)$, and the coefficient of the second term on the rhs has been chosen to give the correct surface integral over $p_{\perp} d p_{\perp} d \phi$ at fixed $|p \cos \theta| \gg p_{0}$. On the string, $\cos \theta= \pm 1$, so that we have two half strings along the two halves of the $\mathbf{e}_{3}$ axis, both taking "magnetic flux" away from the origin. The string term adds exactly $2 \pi$ to the surface integral (83), thereby restoring Stokes theorem and bringing the surface and line integrals into agreement. As mentioned above, gauge transformations with various functional forms $\chi_{\mathbf{p}}(\theta, \phi)$ can shift the strings and replace them by explicitly nonintegrable functions. The choice of $\chi_{\mathbf{p}}^{(1)}$ makes a nonsingular over the integrated surface in Eq. (83) between the loop and the upper pole $\theta=0$, but $\mathbf{a}^{(0)}$ has a singularity penetrating the same surface, giving both line and surface integrals for the phase difference $-\kappa\left(\Delta \chi-\Delta \chi_{\mathbf{p}}\right)$ that differ by $\kappa \Delta \chi_{\mathbf{p}}^{(1)}$. The agreement also extends to use of the surface that includes the pole at

$\theta=\pi$ for both cases 0 and 1 . For the choice $\chi_{\mathbf{p}}^{(2)}$, the line and surface integrals both vanish if the branch cut is inserted explicitly in the surface integral, so that the nonintegrable function in Eq. (54) is replaced by

$$
\mathbf{a}^{(2)}=\frac{\hat{\boldsymbol{\theta}}}{p}\left[\phi-2 \pi h\left(\phi-\phi_{0}\right)\right] \sin \theta,
$$

for example, with $0<\phi_{0}<2 \pi$ where $h\left(\phi-\phi_{0}\right)$ is the Heaviside step function.

\section{DISCUSSION}

In this Section, the nonexistence proofs and recent papers concerning localized states are briefly reviewed, the new photon position operators are discussed in the context of this literature, and our results are summarized. 
The most quoted paper is that of Newton and Wigner [13]. These authors assumed a rotationally invariant set of localized states and arrived at the position operator of the form $i p^{\alpha} \nabla p^{-\alpha}$ for spinless particles with or without mass. They also obtained an expression of the position operator for massless particles of spin $\frac{1}{2}$. Regarding photons, they stated that for $S=1$ and higher "we found that no localized states in the above sense exist. This is an unsatisfactory, if not unexpected, feature of our work." As a result of their conclusion, it is frequently stated that a (spatial) photon wave function does not exist [2]. The localization postulate adopted by Newton and Wigner is strong (any displacement of a localized state is assumed to make it orthogonal to states of the undisplaced set) and has been the focus of a number of more recent studies. In particular, Wightman and others [14] have used generalized imprimitivities [45] to reformulate localization more rigorously in terms of localizability in a region. However, their work did not alter the conclusion that a single photon is not localizable. Other authors have sought effective wave functions that satisfy a somewhat relaxed localization condition [5, 18, 27,46,47.

We suggest a different potential problem with the conclusion of Newton and Wigner for massless particles of spin $S \geq 1$. To ensure a rotationally invariant linear manifold of localized states for a system with total angular momentum quantum number $j$, they assumed a complete set of $2 j+1$ wave functions $\psi_{j m},-j \leq m \leq j$, where $m$ is a component referenced to an external direction. While the existence of a complete set is sufficient to give a rotationally invariant manifold, it is not necessary for massless particles of spin $S>\frac{1}{2}$. Massless particles with spin have only two spin states, namely those corresponding to the helicities $\pm S$. For a system of states at the coordinate origin, the orbital angular momentum vanishes and $j=S$. The states in the linear manifold are characterized by components of j not along a space-fixed direction but along the momentum direction $\hat{\mathbf{p}}$. For $S>\frac{1}{2}$, the manifold is not complete and consequently it cannot describe a state with spin quantized along an arbitrary direction. However, it can describe the allowed states with either helicity. Furthermore, since the helicity operator commutes with the generator $\mathbf{J}$ of rotations, the two helicity subspaces are separately rotationally invariant. Because the helicity eigenstates 
form a complete rotational set only for $S \leq \frac{1}{2}$, it is clear why the Newton's and Wigner's insistence on a complete rotational manifold is stronger than necessary for massless particles with $S \geq 1$.

In the papers based on the method of generalized imprimitivities [14], it appears that the system of commuting imprimitivities, through which the position operator is defined, is assumed to be independent of spin. (This arises from Wightman's axiom V [14]). As seen above, for example in Eq. (46), the spin is inextricably coupled to the momentum and thereby to the position operator. Position operators are known to exist for massless particles of spin 0 , and it may be possible to find a system of imprimitivities like that for the scalar case for each value of the invariant helicity. In any case, the imprimitivities must be distinct for different helicities because the position operators are.

In several more recent proofs of the nonexistence of a photon position operator with commuting components, operator algebra was used with the assumption that the position operators satisfy Eq.(3) [15]18]. However, we have shown above and in Appendix C that the matrix $\underline{\mathbf{r}}$ includes a "gauge potential" a that is transformed by rotations. As a result, the commutator $\left[J_{j}, r_{k}\right]$ contains an additional term involving the spin, and indeed that this extra term is required in order to give the correct phase of the rotated photon state. The components of the position operators (40) thus satisfy Eq.(67), which unlike the more familiar relation (3), is compatible with commuting components. Thus, nonexistence proofs that assume Eq. (3) do not apply.

Our construction of the photon position operator uses a spin rotation to decouple the spin from the momentum while the gradient operator acts to generate momentum-space translations. It seems to be the most natural way to retain the usual role of the position operator in momentum space. While we have not touched on important questions about the limits to which the photon position is observable in a dynamic measurement, our demonstration that a photon position operator does in fact exist means that there is no nonvanishing commutator $\left[\underline{r}_{j}, \underline{r}_{k}\right]$ to limit the calculation of photon probability amplitudes. Simultane- 
ous eigenvectors of the operators $\underline{r}_{j}$ are available for calculation of the probability that the corresponding eigenvalue is observed. Of course limitations arising from Fourier analysis, similar to those applicable to massive particles, still apply. Our work therefore supports the view that photons have wave functions that are not qualitatively different from those of massive particles, as concluded by Białynicki-Birula [46] and Sipe [5]. The rules of quantum mechanics require that each observable be represented by a Hermitian operator. The localized basis sets found here makes it possible to treat photons like massive particles in quantum calculations of interference experiments and other situations where particle amplitudes in coordinate space are useful; in both cases the usual rules of quantum mechanics can be applied.

There is no unique representation of the photon position operator. Just as there are many spin bases that can be used to describe the internal state of a massive particle, there are many bases that can be used to describe the combined translational and internal motion of a photon. The peculiar aspect of the photon is that, because of the coupling of the spin to the momentum, the position operator is a matrix that does not commute with the spin. Different position operators with commuting components can be defined by specifying the dependence $\chi_{\mathbf{p}}(\theta, \phi)$ of the axial rotation angle on the polar angles of $\hat{\mathbf{p}}$. Different choices of $\chi_{\mathbf{p}}$ lead to different "gauge potentials" a for the phase of the photon state at different positions in momentum space. In general, phase changes depend on the path and are thus described by a nonintegrable function. While a specific choice of gauge usually results in operator expressions with less than the expected symmetry of the Hamiltonian, the symmetry is obtained within the full group of possible gauges. The forms that result from a selection of $\chi_{\mathbf{p}}$ are all unitarily equivalent to each other and to the position operator for a massive particle, $i p^{\alpha} \nabla p^{-\alpha}$, where the unitary operator is the rotation through Euler angles. The operator $i p^{\alpha} \nabla p^{-\alpha}$ has eigenvectors with a fixed direction in space and is independent of the spin. Consequently, for massive particles the description of spin is a separable problem. However, the spin and momentum of a photon are inexorably coupled, since the direction of $\mathbf{p}$ determines the direction of the observable component $\hat{\mathbf{p}} \cdot \underline{\mathbf{S}}$ of the internal angular momentum. 
Position operators with transverse and longitudinal eigenvectors could in principle be used to describe a massive particle, but this is probably not useful since $i p^{\alpha} \nabla p^{-\alpha}$ is a simpler alternative. For a massless particle this choice does not exist, reflecting the fact that for a photon, the orbital and spin angular momenta are not separable.

In summary, the arguments presented here show that a photon is much like any other particle in that its position is an observable described by a set of three commuting Hermitian operators. However, the photon (as well as other massless particles of spin $S>\frac{1}{2}$ ) has only two linearly independent spin states, and in these states the spin is coupled to the momentum. As a result, its position operator is a matrix that does not commute with the spin. Different selections of the function $\chi_{\mathbf{p}}(\theta, \phi)$ generally give different position operators, so that the position operator is not unique and does not transform under $\mathbf{J}$ as a simple vector. However, the eigenvectors of any one of these unitarily equivalent position operators gives a basis of localized states with unique eigenvalues that are independent of helicity, and

there is consequently no disagreement as to the actual position of the photon. Contrary to the traditional view, localized basis sets do exist, and it appears that photon wave functions can be defined according the usual rules of quantum mechanics.

\section{ACKNOWLEDGMENTS}

The authors wish to thank the Natural Sciences and Engineering Research Council of Canada for financial support. It is also a pleasure to acknowledge stimulating and helpful communication from B.-S. Skagerstam. One of us (W. E. B.) thanks A. Lasenby and the Astrophysics Group of the Cavendish Laboratory, University of Cambridge, for hospitality during a sabbatical leave taken there, and he thanks J. P. Crawford for helpful discussions of string singularities. 


\section{APPENDIX A: DECOMPOSITION OF ROTATIONS}

The purpose of this appendix is to derive relations between an infinitesimal rotation about an arbitrary axis in three-dimensional space and angular parameters in a product of rotations around specified axes. These relations are then used to predict rotational properties of the photon position operator and its eigenstates. The angular parameters are the Euler angles that specify the polar and azimuthal coordinates $\theta$ and $\phi$, respectively, of a given direction $\hat{\mathbf{p}}$ together with an axial angle $\chi$ about $\hat{\mathbf{p}}$. One can express the Euler-angle parametrization in terms of active rotations about spaced-fixed axes: with the $\mathbf{p}$-frame axes initially coincident with the space-fixed axes $\mathbf{e}_{1}, \mathbf{e}_{2}, \mathbf{e}_{3}$, of the lab frame, a first rotation by $\chi$ about $\mathbf{e}_{3}$ is followed by a rotation by $\theta$ about $\mathbf{e}_{2}$ and finally a rotation by $\phi$ about $\mathbf{e}_{3}$. The axial rotation angle $\chi$ does not affect the direction $\hat{\mathbf{p}}$ and may be chosen to be a function, say $\chi_{\mathbf{p}}(\theta, \phi)$, of the

"local coordinates" $\theta, \phi$ that specify $\hat{\mathbf{p}}$. A rotation by the Euler angles $\left(\phi, \theta, \chi_{\mathbf{p}}(\theta, \phi)\right)$ has only two degrees of freedom and is uniquely determined by the direction $\hat{\mathbf{p}}$.

The derivation is easily constructed using tools of Clifford's geometric algebra $\mathrm{Cl}_{3}$ of 3 dimensional space 48 50. Those not familiar with the algebra can follow the derivation in terms of the $2 \times 2$ matrices that form the common matrix representation in which the Cartesian unit vectors $\mathbf{e}_{1}, \mathbf{e}_{2}, \mathbf{e}_{3}$, are replaced by the corresponding Pauli spin matrices familiar to physicists. In the algebra, vectors are rotated by transformations of the form

$$
\mathbf{v} \rightarrow R \mathbf{v} R^{\dagger}
$$

where $R(\boldsymbol{\xi})=\exp (-i \boldsymbol{\xi} / 2) \in S U(2)$ is the element for a rotation by the angle $\xi=|\boldsymbol{\xi}|$ about the axis $\hat{\xi}$. The rotation specified by the Euler angles $(\phi, \theta, \chi)$ is given by the rotation element

$$
\begin{aligned}
\mathcal{R}(\phi, \theta, \chi) & \equiv R\left(\phi \mathbf{e}_{3}\right) R\left(\theta \mathbf{e}_{2}\right) R\left(\chi \mathbf{e}_{3}\right) \\
& =\exp \left(-i \phi \mathbf{e}_{3} / 2\right) \exp \left(-i \theta \mathbf{e}_{2} / 2\right) \exp \left(-i \chi \mathbf{e}_{3} / 2\right)
\end{aligned}
$$

An additional rotation will generally change all three angular parameters $\phi, \theta, \chi$. We want to determine the changes caused by an infinitesimal rotation $R(d \boldsymbol{\xi})$. 
To clarify our objective, we first consider the simple case in which $d \boldsymbol{\xi}=d \xi \mathbf{e}_{3}$. The only effect of $R(d \boldsymbol{\xi})$ is to increment the azimuthal angle $\phi$ :

$$
\mathcal{R}(\phi, \theta, \chi) \rightarrow R\left(d \xi \mathbf{e}_{3}\right) \mathcal{R}(\phi, \theta, \chi)=\mathcal{R}(\phi+d \xi, \theta, \chi)
$$

This result is easily written in terms of the rotations $\mathcal{R}\left(\phi, \theta, \chi_{\mathbf{p}}(\theta, \phi)\right)$ that have only two degrees of freedom:

$$
\begin{aligned}
& R\left(d \xi \mathbf{e}_{3}\right) \mathcal{R}\left(\phi, \theta, \chi_{\mathbf{p}}(\theta, \phi)\right) \\
= & \mathcal{R}\left(\phi+d \xi, \theta, \chi_{\mathbf{p}}(\theta, \phi+d \xi)\right) R\left(-\mathbf{e}_{3} d \chi_{\mathbf{p}}\right)
\end{aligned}
$$

where $\chi_{\mathbf{p}}(\theta, \phi+d \xi)=\chi_{\mathbf{p}}(\theta, \phi)+d \chi_{\mathbf{p}}$ and $d \chi_{\mathbf{p}}=d \xi \partial \chi_{\mathbf{p}} / \partial \phi$.

Now we generalize this approach to an arbitrary infinitesimal rotation by $d \boldsymbol{\xi}=d \xi_{1} \mathbf{e}_{1}+$ $d \xi_{2} \mathbf{e}_{2}+d \xi_{3} \mathbf{e}_{3}$. We initially consider $\chi$ an independent parameter and solve $R(d \boldsymbol{\xi}) \mathcal{R}(\phi, \theta, \chi)=$ $\mathcal{R}(\phi+d \phi, \theta+d \theta, \chi+d \chi)$ for the infinitesimal changes $d \phi, d \theta, d \chi$ in the Euler angles. To first order in the changes, $\mathcal{R}(\phi+d \phi, \theta+d \theta, \chi+d \chi)$

$$
\begin{aligned}
& =\left[1-\frac{i}{2}\left(\mathbf{e}_{3} d \phi+e^{-i \mathbf{e}_{3} \phi} \mathbf{e}_{2} d \theta+R \mathbf{e}_{3} R^{\dagger} d \chi\right)\right] \mathcal{R}(\phi, \theta, \chi) \\
& =\left\{1-\frac{i}{2}\left[\mathbf{e}_{1}(\sin \theta \cos \phi d \chi-\sin \phi d \theta)+\mathbf{e}_{2}(\cos \phi d \theta\right.\right. \\
& \left.\left.+\sin \theta \sin \phi d \chi)+\mathbf{e}_{3}(d \phi+\cos \theta d \chi)\right]\right\} \mathcal{R}(\phi, \theta, \chi),
\end{aligned}
$$

in which we have explicitly accounted for the lack of commutivity of rotations about different axes. Equation (A4) therefore implies

$$
\begin{aligned}
d \boldsymbol{\xi}= & \mathbf{e}_{1}(\sin \theta \cos \phi d \chi-\sin \phi d \theta) \\
& +\mathbf{e}_{2}(\cos \phi d \theta+\sin \theta \sin \phi d \chi)+\mathbf{e}_{3}(d \phi+\cos \theta d \chi),
\end{aligned}
$$

which is readily solved to give

$$
\begin{aligned}
d \phi & =d \xi_{3}-\cot \theta\left(d \xi_{1} \cos \phi+d \xi_{2} \sin \phi\right) \\
d \theta & =d \xi_{2} \cos \phi-d \xi_{1} \sin \phi \\
d \chi & =\frac{d \xi_{1} \cos \phi+d \xi_{2} \sin \phi}{\sin \theta} .
\end{aligned}
$$


Note the singular nature of the relations for $d \phi$ and $d \chi$ in the limit $\theta \rightarrow 0$. Singularities are common whenever general rotations are parametrized in terms of rotations about specified axes. They are related to the nonuniqueness of the parametrization for some rotations, for example all of the rotations $D(\phi, 0, \chi-\phi)$ for fixed $\chi$ and arbitrary $\phi$ are equal. We can also express the rotation (A5) in terms of rotations with two degrees of freedom

$$
\begin{aligned}
& R(d \boldsymbol{\xi}) \mathcal{R}\left(\phi, \theta, \chi_{\mathbf{p}}\right)=\mathcal{R}\left(\phi+d \phi, \theta+d \theta, \chi_{\mathbf{p}}+d \chi\right) \\
& =\mathcal{R}\left(\phi+d \phi, \theta+d \theta, \chi_{\mathbf{p}}+d \chi_{\mathbf{p}}\right) R\left(\left(d \chi-d \chi_{\mathbf{p}}\right) \mathbf{e}_{3}\right) \\
& =R\left(\left(d \chi-d \chi_{\mathbf{p}}\right) \hat{\mathbf{p}}\right) \mathcal{R}\left(\phi+d \phi, \theta+d \theta, \chi_{\mathbf{p}}+d \chi_{\mathbf{p}}\right)
\end{aligned}
$$

where $\chi_{\mathbf{p}}=\chi_{\mathbf{p}}(\theta, \phi)$ and

$$
\begin{aligned}
d \chi-d \chi_{\mathbf{p}} & =d \chi-\left(\frac{\partial \chi_{\mathbf{p}}}{\partial \phi} d \phi+\frac{\partial \chi_{\mathbf{p}}}{\partial \theta} d \theta\right) \\
& =\left[\frac{\cos \phi}{\sin \theta}\left(1+\cos \theta \frac{\partial \chi_{\mathbf{p}}}{\partial \phi}\right)+\sin \phi \frac{\partial \chi_{\mathbf{p}}}{\partial \theta}\right] d \xi_{1} \\
& +\left[\frac{\sin \phi}{\sin \theta}\left(1+\cos \theta \frac{\partial \chi_{\mathbf{p}}}{\partial \phi}\right)-\cos \phi \frac{\partial \chi_{\mathbf{p}}}{\partial \theta}\right] d \xi_{2} \\
& -\frac{\partial \chi_{\mathbf{p}}}{\partial \phi} d \xi_{3} \\
& =(\mathbf{a} \times \mathbf{p}+\hat{\mathbf{p}}) \cdot d \boldsymbol{\xi}
\end{aligned}
$$

where in the last step we used the definition (44,45) of $\mathbf{a}$. While the derivation employs tools of geometric algebra, the result is generally valid for spatial rotations in three-dimensional space.

A similar analysis gives the result of an infinitesimal rotations added to the rhs:

$$
\begin{aligned}
& \mathcal{R}\left(\phi, \theta, \chi_{\mathbf{p}}\right) R\left(d \boldsymbol{\xi}^{\prime}\right)=\mathcal{R}\left(\phi+d \phi, \theta+d \theta, \chi_{\mathbf{p}}+d \chi\right) \\
& =\mathcal{R}\left(\phi+d \phi, \theta+d \theta, \chi_{\mathbf{p}}+d \chi_{\mathbf{p}}\right) R\left(\left(d \chi-d \chi_{\mathbf{p}}\right) \mathbf{e}_{3}\right)
\end{aligned}
$$


In this case, we obtain

$$
\begin{aligned}
d \phi & =\frac{-d \xi_{1}^{\prime} \cos \chi+d \xi_{2}^{\prime} \sin \chi}{\sin \theta} \\
d \theta & =d \xi_{1}^{\prime} \sin \chi+d \xi_{2}^{\prime} \cos \chi \\
d \chi & =d \xi_{3}^{\prime}-d \phi \cos \theta \\
d \chi-d \chi_{\mathbf{p}} & =d \xi_{1}^{\prime}\left[\frac{\cos \chi}{\sin \theta}\left(\cos \theta+\frac{\partial \chi_{\mathbf{p}}}{\partial \phi}\right)-\sin \chi \frac{\partial \chi_{\mathbf{p}}}{\partial \theta}\right] \\
& -d \xi_{2}^{\prime}\left[\frac{\sin \chi}{\sin \theta}\left(\cos \theta+\frac{\partial \chi_{\mathbf{p}}}{\partial \phi}\right)+\cos \chi \frac{\partial \chi_{\mathbf{p}}}{\partial \theta}\right] \\
& +d \xi_{3}^{\prime} .
\end{aligned}
$$

These results can also be obtained from the Hermitian conjugate of the relations (A5) and (A4) by noting that $D^{\dagger}(\phi, \theta, \chi)=D(-\chi,-\theta,-\phi)$. Such added rotations from the rhs are rotations in the $\mathbf{p}$-frame and can also be expressed by

$$
\mathcal{R}\left(\phi, \theta, \chi_{\mathbf{p}}\right) R\left(d \xi_{j}^{\prime} \mathbf{e}_{j}\right)=R\left(d \xi_{j}^{\prime} \mathbf{e}_{\mathbf{p} j}\right) \mathcal{R}\left(\phi, \theta, \chi_{\mathbf{p}}\right)
$$

Evidently the Cartesian components of $d \boldsymbol{\xi}^{\prime}$ are just the $\mathbf{p}$-frame components of $d \boldsymbol{\xi}$, that is, $d \xi_{j}^{\prime}=d \xi_{\mathbf{p} j}$. We make use of this result in Appendix D.

\section{APPENDIX B: APPLICATION TO ROTATIONS OF STATE VECTORS}

The helicity state vectors $\underline{\mathbf{e}_{\mathbf{p} \kappa}}$ are obtained by rotating the constant column vectors $\underline{\mathbf{e}_{\kappa}}$

$$
\underline{\mathbf{e}_{\mathbf{p} \kappa}}=\underline{D}(\phi, \theta, \chi) \underline{\mathbf{e}_{\kappa}},
$$

by the rotation matrix

$$
\underline{D}=\exp \left(-i \underline{S_{3}} \phi\right) \exp \left(-i \underline{S_{2}} \theta\right) \exp \left(-i \underline{S_{3}} \chi\right)
$$

generated by the spin-one matrices $S_{j}$. Starting in the Cartesian basis with

$$
\underline{\mathbf{e}_{0}}=\left(\begin{array}{c}
0 \\
0 \\
1
\end{array}\right), \underline{\mathbf{e}_{ \pm 1}}=\frac{1}{\sqrt{2}}\left(\begin{array}{c}
1 \\
\pm i \\
0
\end{array}\right)
$$


the rotation matrix is explicitly

$$
D=\left(\begin{array}{ccc}
\cos \theta \cos \phi \cos \chi-\sin \phi \sin \chi & -(\sin \phi \cos \chi+\cos \theta \cos \phi \sin \chi) & \sin \theta \cos \phi \\
\cos \theta \sin \phi \cos \chi+\cos \phi \sin \chi & \cos \phi \cos \chi-\cos \theta \sin \phi \sin \chi & \sin \theta \sin \phi \\
-\sin \theta \cos \chi & \sin \theta \sin \chi & \cos \theta
\end{array}\right)
$$

and we thus find

$$
\begin{aligned}
& \underline{\mathbf{e}_{\mathbf{p} 0}}=\left(\begin{array}{c}
\sin \theta \cos \phi \\
\sin \theta \sin \phi \\
\cos \theta
\end{array}\right), \\
& \underline{\mathbf{e}_{\mathbf{p} \kappa}}=\frac{e^{-i \kappa \chi}}{\sqrt{2}}\left(\begin{array}{c}
\cos \theta \cos \phi-i \kappa \sin \phi \\
\cos \theta \sin \phi+i \kappa \cos \phi \\
-\sin \theta
\end{array}\right), \kappa= \pm 1 .
\end{aligned}
$$

Note that $\underline{\mathbf{e}_{\mathbf{p} 0}}$ is a matrix representation of $\hat{\mathbf{p}}$, and $\underline{\mathbf{e}_{\mathbf{p} \kappa}}$ depends on $\chi$ simply through the phase factor $e^{-i \kappa \chi}$. We put $\chi=\chi_{\mathbf{p}}(\theta, \phi)$ to obtain vectors $\underline{\mathbf{e}_{\mathbf{p} \kappa}}$ that depend only on the two angles $\theta$ and $\phi$, and hence only on the direction of $\hat{\mathbf{p}}$. The direction of $\hat{\mathbf{p}}$ can be changed by a further rotation of $\underline{\mathbf{e}_{\mathbf{p} \kappa}}$ by $d \boldsymbol{\xi}$ of the form $\exp (-i d \boldsymbol{\xi} \cdot \underline{\mathbf{S}}) \underline{\mathbf{e}_{\mathbf{p} \kappa}}$

$$
\begin{aligned}
& =\underline{D}\left(\phi+d \phi, \theta+d \theta, \chi_{\mathbf{p}}+d \chi\right) \underline{\mathbf{e}_{\kappa}} \\
& =\exp \left[-i \kappa\left(d \chi-d \chi_{\mathbf{p}}\right)\right] \underline{D}\left(\phi+d \phi, \theta+d \theta, \chi_{\mathbf{p}}+d \chi_{\mathbf{p}}\right) \underline{\mathbf{e}_{\kappa}} \\
& =\exp \left[-i \kappa\left(d \chi-d \chi_{\mathbf{p}}\right)\right] \underline{\mathbf{e}_{\mathbf{p}+d \mathbf{p} \kappa}} .
\end{aligned}
$$

The rotation matrix $\exp (-i d \boldsymbol{\xi} \cdot \underline{\mathbf{S}})$ shuffles the components of the vector $\underline{\mathbf{e}_{\mathbf{p} \kappa}}$. A complete rotation of $\underline{\mathbf{e}_{\mathbf{p} \kappa}}$ also includes the operator $\exp (-i d \boldsymbol{\xi} \cdot \boldsymbol{L})$ which changes the angular arguments $\theta, \phi$ so as to rotate $\hat{\mathbf{p}}$ to $\hat{\mathbf{p}}-d \hat{\mathbf{p}}$. We thus find $\exp (-i d \boldsymbol{\xi} \cdot \underline{\mathbf{J}}) \underline{\mathbf{e}_{\mathbf{p} \kappa}}$

$$
\begin{aligned}
& =\exp \left[-i \kappa\left(d \chi-d \chi_{\mathbf{p}}\right)\right] \exp (-i d \boldsymbol{\xi} \cdot \boldsymbol{L}) \underline{\mathbf{e}_{\mathbf{p}+d \mathbf{p} \kappa}} \\
& =\exp \left[-i \kappa\left(d \chi-d \chi_{\mathbf{p}}\right)\right] \underline{\mathbf{e}_{\mathbf{p} \kappa}}
\end{aligned}
$$

and consequently the only effect of a rotation of $\underline{\mathbf{e}_{\mathbf{p} \kappa}}$ by $d \xi$ is to change its phase by $-\kappa\left(d \chi-d \chi_{\mathbf{p}}\right)$, where $d \chi-d \chi_{\mathbf{p}}$ is given by Eq. (A10). This agrees with the partition 
of $\underline{\mathbf{J}}$ into

$$
\underline{\mathbf{J}}=\underline{\mathbf{L}}^{(r)}+\underline{\mathbf{S}}^{(r)}
$$

since

$$
\underline{\mathbf{L}}^{(r)} \underline{\mathbf{e}_{\mathbf{p} \kappa}}=\underline{D} \mathbf{L} \underline{D}^{-1} \underline{\mathbf{e}_{\mathbf{p} \kappa}}=\underline{D} \mathbf{L} \underline{\mathbf{e}_{\kappa}}=0
$$

and

$$
\begin{aligned}
\underline{\mathbf{S}^{(r)}} & =\hat{\mathbf{p}} \cdot \underline{\mathbf{S}}(\mathbf{a} \times \mathbf{p}+\hat{\mathbf{p}}) \\
\hat{\mathbf{p}} \cdot \underline{\mathbf{S}} \underline{\mathbf{e}_{\mathbf{p} \kappa}} & =\underline{D} \underline{S_{3}} \underline{D}^{-1} \underline{\mathbf{e}_{\mathbf{p} \kappa}}=\underline{D} \underline{S_{3}} \underline{\mathbf{e}_{\kappa}}=\kappa \underline{\mathbf{e}_{\mathbf{p} \kappa}} \\
(\mathbf{a} \times \mathbf{p}+\hat{\mathbf{p}}) \cdot d \boldsymbol{\xi} & =d \chi-d \chi_{\mathbf{p}} .
\end{aligned}
$$

\section{APPENDIX C: APPLICATION TO ROTATIONS OF THE POSITION OPERATOR}

The new position operator has components

$$
\underline{r_{l}}=i p^{\alpha} \underline{D} \frac{\partial}{\partial p_{l}} \underline{D}^{-1} p^{-\alpha} .
$$

The components of vector operators are expected to rotate into one another as given by the commutation relation (3). However, an additional factor arises by the need to transform the matrix associated with each vector component by an axial rotation. The required spin rotation is given by that for the rotation matrix $\underline{D}$. From relation (A8) we see that the rotation transforms $\underline{D}\left(\phi, \theta, \chi_{\mathbf{p}}\right)$ to $(1-i \underline{\mathbf{S}} \cdot d \boldsymbol{\xi}) \underline{D}\left(\phi, \theta, \chi_{\mathbf{p}}\right)$

$$
\begin{aligned}
& =\underline{D}\left(\phi+d \phi, \theta+d \theta, \chi_{\mathbf{p}}+d \chi_{\mathbf{p}}\right)\left(1-i \underline{S_{3}}\left(d \chi-d \chi_{\mathbf{p}}\right)\right) \\
& =\left(1-i \hat{\mathbf{p}} \underline{\mathbf{S}}\left(d \chi-d \chi_{\mathbf{p}}\right)\right) \underline{D}\left(\phi+d \phi, \theta+d \theta, \chi_{\mathbf{p}}+d \chi_{\mathbf{p}}\right),
\end{aligned}
$$

where from Eqs. (A11) and (A10),

$$
\hat{\mathbf{p}} \cdot \underline{\mathbf{S}}\left(d \chi-d \chi_{\mathbf{p}}\right)=\hat{\mathbf{p}} \cdot \underline{\mathbf{S}} d \boldsymbol{\xi} \cdot(\hat{\mathbf{p}}+\mathbf{a} \times \mathbf{p})=\underline{\mathbf{S}}^{(r)} \cdot d \boldsymbol{\xi} .
$$

The result is to add $\left[\underline{S}_{j}^{(r)}, \underline{r_{k}}\right]$ to the rhs of Eq. (31). The added term is required for consistency with the phase changes (B10) induced in the basis vectors by rotations. 


\section{APPENDIX D: ROTATIONS OF THE REFERENCE FRAME AND BERRY'S PHASE}

An extra rotation acting on the rhs of $R(\phi, \theta, \chi)$ is equivalent to the opposite rotation to the reference frame. As shown in relation (A16), it is also equivalent to a rotation from the left by the same angles but about $\mathbf{p}$-frame axes instead of lab axes. This relation is useful in deriving an expression for Berry's phase [34]. Berry's phase is a topological phase that arises from adiabatic transport of a discrete state around a closed loop, and it is usually derived as a purely quantum phenomenon [34, 13, [4]]. For the polarized light, however, it was shown by Haldane [51] to be a consequence of classical differential geometry. We derive it here by means of the classical rotation operators introduced above.

Transport in the adiabatic limit corresponds to parallel transport in the given gauge [35.52], which is equivalent to nonrotating (Fermi-Walker) transport on geodesics. We achieve this in rotations of $\mathbf{p}$ by piecing together the closed loop from a sequence of greatcircle rotations, in each of which the rotation axis is orthogonal to $\mathbf{p}$ and the axial rotation vanishes. Thus, in Eq. (A8) we vary $\xi^{\prime}$ such that $d \xi_{3}^{\prime}=0$. Then from Eq. (A14),

$$
d \chi=-\cos \theta d \phi
$$

We vary the parameters $\xi_{1}^{\prime}$ and $\xi_{2}^{\prime}$ so as to complete a closed loop, increasing $\phi$ by $2 \pi$. The condition $d \xi_{3}^{\prime}=0$ ensures that the change in $\hat{\mathbf{p}}$ is always along a great circle. There is a change in the axial angle $-\chi$ by

$$
-\Delta \chi=\oint \cos \theta d \phi
$$

even after $\mathbf{p}$ has looped back to its original direction. If the initial orientation of the photon frame is given by $R(\phi, \theta, \chi)$ with the momentum direction $\hat{\mathbf{p}}$, the orientation after $\phi$ has increased by $2 \pi$ is $R(\phi+2 \pi, \theta, \chi+\Delta \chi)$, giving a the net rotation of

$$
\begin{aligned}
& R(\phi+2 \pi, \theta, \chi+\Delta \chi) R^{-1}(\phi, \theta, \chi) \\
= & \exp [-i(\chi+\Delta \chi) \hat{\mathbf{p}} / 2] \exp \left(-i \pi \mathbf{e}_{3}\right) \exp (i \chi \hat{\mathbf{p}} / 2) \\
= & \exp [-i(\Delta \chi+2 \pi) \hat{\mathbf{p}} / 2],
\end{aligned}
$$


where we used the symmetry

$$
R(\phi, \theta, \chi)=\exp (-i \chi \hat{\mathbf{p}} / 2) R(\phi, \theta, 0)
$$

which allows us to cancel the $\theta$ rotations, and we noted that $\exp \left(-i \pi \mathbf{e}_{3}\right)=-1=$ $\exp (-i \pi \hat{\mathbf{p}})$. The total rotation under parallel transport about the loop is thus the rotation about $\hat{\mathbf{p}}$ by the angle $\eta=\Delta \chi+2 \pi=\oint(1-\cos \theta) d \phi$.

Note that the result does depend on the parallel transport of $\mathbf{p}$. If we rotate $\mathbf{p}$ directly around the space-fixed $\mathbf{e}_{3}$ axis, then we simply change $\phi$ by $2 \pi$ and the axial angle $\chi$ does not change. The mathematical confirmation of this result is found from Eq. (A7) with $d \xi_{1}=d \xi_{2}=0$. The rotation in this case is not on a great circle (unless $\cos \theta=0$ ) and $\mathbf{p}$ is therefore not parallel transported.

The phase change for electromagnetic plane waves of given helicity is proportional to the rotation angle. To see the relation, consider electromagnetic plane waves of helicity $\kappa$, for which the field $\mathbf{F}=\mathbf{E}+i c \mathbf{B}$ is 49

$$
\mathbf{F}=(1+\hat{\mathbf{p}}) \mathbf{E}_{0} \exp [i \kappa(\omega t-\mathbf{p} \cdot \mathbf{x}) \hat{\mathbf{p}}]
$$

An additional axial rotation by $\eta$ transforms this to

$$
\begin{aligned}
& \exp (-i \eta \hat{\mathbf{p}} / 2) \mathbf{F} \exp (i \eta \hat{\mathbf{p}} / 2)=\mathbf{F} \exp (i \eta \hat{\mathbf{p}}) \\
= & (1+\hat{\mathbf{p}}) \mathbf{E}_{0} \exp [i \kappa(\omega t-\mathbf{p} \cdot \mathbf{x}+\kappa \eta) \hat{\mathbf{p}}]
\end{aligned}
$$

and gives the phase change $-\kappa \eta$ relative to $-\omega t$. This is exactly Berry's phase (80)

$$
\kappa \oint(\cos \theta-1) d \phi=-\kappa \Omega
$$

where $\Omega$ is the solid angle subtended by the loop. It is just that calculated directly from the line integral in Section $\mathrm{V}$ when $\chi_{\mathbf{p}}=\chi_{\mathbf{p}}^{(1)}=-\phi$ so that the rotation matrix $D\left(\phi, \theta, \chi_{\mathbf{p}}\right)$ rotates $S_{3}$ directly into $\mathbf{S} \cdot \hat{\mathbf{p}}$, and it agrees with the relation between angle and geometric phase found by Berry [53]. 


\section{REFERENCES}

* email: margaret.hawton.lakeheadu.ca

$\dagger \quad$ email: baylis.uwindsor.ca

[1] W. Pauli, General Principles of Quantum Mechanics (Springer-Verlag, New York, 1980; translation of Prinzipien der Quantentheorie, Handbuch der Physik, Vol.24, Springer, Berlin, 1933).

[2] C. Cohen-Tannoudji,, J. Dupont-Roc and G. Grynberg, Photons and Atoms: Introduction to Quantum Electrodynamics (John Wiley and Sons, New York, 1989), pp.30, 50, 188.

[3] A.I. Akhiezer and V.B. Berestetskii, Quantum Electrodynamics (Interscience, New York, 1965).

[4] See for example, I. Białynicki-Birula, "Photon Wave Function" in Progress in Optics, Vol. 36, ed. E. Wolf (Elsevier, Amsterdam, 1996) and references therein.

[5] J. E. Sipe, Phys. Rev. A 52, 1875 (1995).

[6] G. N. Fleming, Phys. Rev. 137, B188 (1965).

[7] G. N. Fleming, J. Math. Phys. 7, 1959 (1966).

[8] G. C. Hegerfeldt, Phys. Rev. D 10, 3320 (1974).

[9] B.-S. Skagerstam, Int. J. Theor. Phys. 15, 213 (1976).

[10] W. Cegła and B. Jancewicz, Rep. Math. Phys. 11, 53 (1977).

[11] B. Jancewicz, J. Math. Phys. 18, 2487 (1977).

[12] G. C. Hegerfeldt, Phys. Rev. Lett. 54, 2395 (1985).

[13] T.D. Newton and E.P. Wigner, Rev. Mod. Phys. 21, 400 (1949). 
[14] A.S. Wightman, Rev. Mod. Phys. 34, 845 (1962); J.M. Jauch and C. Piron, Helv. Phys. Acta 40, 559 (1967); W.O. Amrein, Helv. Phys. Acta 42, 149 (1969); E. R. Pike and S. Sarkar, The Quantum Theory of Radiation (Clarendon Press, Oxford 1995).

[15] T.F. Jordan and N. Mukunda, Phys. Rev. 132, 1842 (1963).

[16] A. Z. Jadczyk and B. Jancewicz, Bull. Acad. Pol., Ser. Math. Astr. Phys. 21, 477 (1973).

[17] T.F. Jordan, J. Math. Phys. 21, 1382 (1978).

[18] J. Mourad, Phys. Lett. A182, 319 (1993).

[19] M. Hawton, Phys. Rev. A 59, 954 (1999), referred to as I. Note that what was denoted by $\mathbf{a}$ in $\mathrm{I}$ is $\mathbf{a}(0) \hat{\mathbf{p}} \cdot \mathbf{S}$ in the current paper.

[20] E. Wigner, Annals of Mathematics 40, 149 (1939).

[21] V. Bargman and E.P. Wigner, Proc. Natl. Acad. Sci. 34, 211 (1948).

[22] M.H.L. Pryce, Proc. Roy. Soc. 195A, 62 (1948).

[23] L.L. Foldy, Phys. Rev. 102, 568 (1956).

[24] A. Chakrabarti, J. Math. Phys. 7, 949 (1966).

[25] J.S. Lomont and H.E. Moses, J. Math. Phys. 3, 405 (1962).

[26] Iu.M. Shirokov, Sov. Phys. JETP 6, 919 (1958).

[27] I. Białynicki-Birula and Z. Białynicka-Birula, Quantum Electrodynamics (Pergamon, Warsaw, 1975).

[28] I. Białynicki-Birula and Z. Białynicka-Birula, Phys. Rev. D 35, 2383 (1987), Sec. 9. Note that to make the matrix $W_{1}$ of the authors' Eq. (A14b) unitary, its middle column should be multiplied by $\sqrt{2}$, and in Eq. (10d) the factor $\left(E_{p}+m\right)$ evidently should be raised to the -1 power. 
[29] D. M. Brink and G. R. Satchler, Angular Momentum, 3rd edn. (Oxford University Press, Oxford, 1993).

[30] M. Hawton, Phys. Rev. A 59, 3223 (1999).

[31] A. D. Fokker, Relativiteitstheorie (Noordhoff, Groningen, 1929).

[32] B.-S. Skagerstam, in On Klauders Path: A Field Trip. Festschrift for John R. Klauder on Occasion of His 60th Birthday, Eds. G. G. Emch, G. C. Hegerfeldt and L. Streit (World Scientific, Singapore 1994), p. 209.

[33] B.-S. Skagerstam, Topics in Modern Quantum Optics. Lectures presented at the 17th Symposium on Theoretical Physics - Applied Field Theory (Seoul National University, Seoul, Korea, 1998).

[34] M.V. Berry, Proc, R. Soc. Lond. A392, 45 (1984).

[35] R.Y. Chiao and Y.-S. Wu, Phys. Rev. Lett. 57, 933 (1986); A. Tomita and R.Y. Chiao, Phys. Rev. Lett. 57, 937 (1986); 59, 1789 (1987); P. G. Kwiat and R. Y. Chiao, Phys. Rev. Lett. 66, 588 (1991).

[36] A. Staruszkiewicz, Int. J. Theor. Phys. 8, 247 (1973); A. Staruszkiewicz, Acta Phys. Pol. B4, 57 (1973).

[37] A. P. Balachandran, G. Marmo, B.-S. Skagerstam and A. Stern, Gauge Symmetries and Fibre Bundles: Applications to Particle Dynamics (Springer Verlag, Berlin 1983), Section 5.6.

[38] T. T. Wu and C. N. Yang, Phys. Rev. D 12, 3845 (1975).

[39] P. Goddard and D. I. Olive, Rep. Prog. Phys. 41, 1357 (1978).

[40] A. K. Pati, Phys. Lett. A 218, 5 (1996).

[41] B.-S. Skagerstam and A. Stern, Nucl. Phys. B294, 636 (1987). 
[42] T.F. Jordan, J. Math. Phys. 21, 2028 (1980).

[43] Y. Aharonov and J. Anandan, Phys. Rev. Lett. 58, 1593 (1987).

[44] J. Anandan and L. Stodolsky, Phys. Rev. D 35, 2597 (1987).

[45] G.W. Mackey, Proc. Natl. Acad. Sci. U.S. 35, 537 (1949).

[46] I. Białynicki-Birula, Phys. Rev Lett. 80, 5247 (1998).

[47] C. Adlard, E.R. Pine and S. Sarkar, Phys. Rev. Lett. 79, 1585 (1997).

[48] D. Hestenes, Spacetime Algebra, (Gordon and Breach, New York, 1966).

[49] W.E. Baylis, Electrodynamics: A Modern Geometric Approach, Progress in Physics Series 17 (Birkhäuser, Boston, 1999).

[50] P. Lounesto, Clifford Algebras and Spinors (Cambridge University Press, UK, 1997).

[51] F. D. M. Haldane, Opt. Lett. 11, 730 (1986); Phys. Rev. Lett. 59, 1788 (1987).

[52] B. Simon, Phys. Rev. Lett. 51, 2167 (1983).

[53] M. V. Berry, J. Phys. A 18, 15 (1985). 